\title{
Solutions Fondamentales et Estimations Optimales pour l'Opérateur de Cauchy-Riemann Tangentiel
}

\author{
Moulay Youssef Barkatou \& \\ Christine LAURENT-Thiébaut
}

Dans le cadre de l'étude de l'opérateur de Cauchy-Riemann tangentiel sur les variétés Cauchy-Riemann (CR), il est particulièrement intéressant d'obtenir des estimations optimales pour la résolution de l'équation $\bar{\partial}_{b} u=f$ ainsi que des théorèmes de régularité.

Dans cet article nous considérons le cas d'une variété CR générique $q$-concave $M$ de codimension réelle $k$, plongée dans $\mathbb{C}^{n}$ et nous construisons un noyau $R_{M}$ sur $M$, qui possède des propriétés analogues au noyau de Bochner-Martinelli dans $\mathbb{C}^{n}$. Le résultat principal est le théorème suivant:

THÉORÈME 0.1. Soient $M$ une variété CR générique q-concave, de codimension réelle $k$, de classe $\mathcal{C}^{3}$ plongée dans $\mathbb{C}^{n}$ et $z_{0}$ un point de $M$. Il existe un voisinage $U_{z_{0}}$ de $z_{0}$ dans $M$ et un noyau $R_{M}$ tel que si $\Omega$ est un domaine à bord $\mathcal{C}^{1}$ relativement compact dans $M \cap U_{z_{0}}$ alors

(i) Si f est une $(n, r)$-forme de classe $\mathcal{C}^{1}$ dans $\bar{\Omega}, n-k-q+1 \leq r \leq n-k$, on a la formule suivante au sens des courants sur $M$

$$
\begin{aligned}
(-1)^{(k+1)(r+n)+\frac{k(k+1)}{2} f(z)=} & \bar{\partial}_{b} \int_{\zeta \in \Omega} f(\zeta) \wedge R_{M}(z, \zeta) \\
& +(-1)^{k+1} \int_{\zeta \in \Omega} \bar{\partial}_{b} f(\zeta) \wedge R_{M}(z, \zeta) \\
& +(-1)^{k} \int_{\zeta \in \partial \Omega} f(\zeta) \wedge R_{M}(z, \zeta) .
\end{aligned}
$$

(ii) Si f est une $(n, r)$-forme de classe $\mathcal{C}^{1}$ dans $\bar{\Omega}, 0 \leq r \leq q-1$, on a la formule suivante au sens des courants sur $M$

$$
\begin{aligned}
(-1)^{(k+1)(r+n)+\frac{k(k+1)}{2}} f(\zeta)= & \bar{\partial}_{b} \int_{z \in \Omega} f(z) \wedge R_{M}(z, \zeta) \\
& +(-1)^{k+1} \int_{z \in \Omega} \bar{\partial}_{b} f(z) \wedge R_{M}(z, \zeta) \\
& +(-1)^{k} \int_{z \in \partial \Omega} f(z) \wedge R_{M}(z, \zeta)
\end{aligned}
$$

Received June 10, 2005. Revision received September 27, 2005. 
(iii) Les opérateurs intégraux définis par le noyau $R_{M}$ sont continus de $\mathcal{C}_{n, r}^{l}(\bar{\Omega})$ dans $\mathcal{C}_{n, r-1}^{l+1 / 2}(\Omega)$, si $M$ est de classe $\mathcal{C}^{l+2}$ et $n-k-q+1 \leq r \leq n-k$ ou si M est de classe $\mathcal{C}^{l+3}$ et $1 \leq r \leq q$.

On en déduit un Lemme de Poincaré pour l'opérateur de Cauchy-Riemann tangentiel avec régularité $\mathcal{C}^{l+1 / 2}$ (cf. Théorème 5.9).

Le Théorème 0.1 a déjà été annoncé dans [6] mais malheureusement une erreur s'est glissée dans la construction des noyaux (ils ne possèdent pas les propriétés d'holomorphie exigées). Ici nous utilisons les noyaux introduits dans [16] pour résoudre l'équation de Cauchy-Riemann dans des domaines à coins $q$-convexes de $\mathbb{C}^{n}$. La démonstration reprend de nombreux arguments de [6]. Le Théorème 5.9 est prouvé dans [5] en s'appuyant sur les résultats de [6], nous en redonnons une démonstration utilisant les noyaux construits ici et dont les grandes lignes suivent celle de [5].

Un corollaire important est un résultat de régularité pour l'opérateur de CauchyRiemann tangentiel dans un cas où l'équation de Cauchy-Riemann tangentielle n'a pas de solution locale.

Corollaire 0.2. Soient $M$ une sous-variété CR générique 1-concave, de classe $\mathcal{C}^{l+3}$ d'une variété analytique complexe et $T$ une distribution d'ordre l sur $M$. Si $\bar{\partial}_{b} T$ est une $(0,1)$-forme de classe $\mathcal{C}^{l}$ sur $M$ alors $T$ est en fait une fonction de classe $\mathcal{C}^{l+1 / 2}$ sur $M$.

Le Corollaire 0.2 améliore un résultat de [3] (voir également [2, Thm. 1]) où l'auteur prouve un théorème de régularité hölderienne d'ordre $\frac{1}{2}-\varepsilon$ si $M$ est de classe $\mathcal{C}^{3}$ et d'ordre $1 / 2^{k}-\varepsilon$ si $M$ est de classe $\mathcal{C}^{2}$. On ignore actuellement comment éviter la perte de régularité lorsque $M$ est de classe $\mathcal{C}^{2}$, même dans le cas des hypersurfaces. Il est démontré par Fischer [9], lorsque $M$ est une hypersurface. Nous ne répétons pas ici la démonstration, qui est identique à celle donnée dans [9] (voir aussi [3]).

Replaçons maintenant ces résultats dans leur contexte historique. Les premières solutions fondamentales pour l'opérateur de Cauchy-Riemann tangentiel ont été définies vers 1975, dans le cas où $M$ est le bord d'un domaine strictement strictement pseudoconvexe de $\mathbb{C}^{n}$, dans des travaux de Romanov [21], Henkin [12], et Skoda [23]. Les noyaux construits indépendamment par Henkin et Skoda ont été repris par Boggess dans son livre [7], où il prouve des estimations $\mathcal{C}^{l}$. Ensuite Harvey et Polking ont considéré dans [11] le cas des hypersurfaces faiblement pseudoconvexes possédant une fonction support birégulière.

Lorsque $M$ est une hypersurface dont la forme de Levi possède une signature mixte, la condition naturelle sur $M$ pour obtenir des résultats de résolubilité locale pour l'équation de Cauchy-Riemann tangentielle est la condition $Y(q)$ de Kohn. En 1985, Boggess et Shaw [BoSh] ont défini des noyaux permettant de résoudre l'équation de Cauchy-Riemann tangentielle dans ce cadre pour une donnée à support compact. En 1992, Fischer et Leiterer [10] ont prouvé une formule de Bochner-Martinelli-Koppelman pour les hypersurfaces de classe $\mathcal{C}^{2}$ dont la forme 
de Levi possède $q$ paires de valeurs propres de signes opposés, ainsi que des estimations uniformes. Dans [4], le premier auteur a amélioré leurs résultats en prouvant des estimations $\mathcal{C}^{1 / 2-\varepsilon}, \varepsilon>0$, pour les mêmes noyaux. Finalement Fischer [9] a construit de nouveaux noyaux permettant d'obtenir les estimations optimales $\mathcal{C}^{l+1 / 2}$ dans ce cadre. Notons également le travail [22] de Shaw qui étend les résultats de Henkin aux hypersurfaces satisfaisant la condition $Y(q)$ et construit une solution fondamentale pour l'opérateur de Cauchy-Riemann tangentiel sur $M$.

L'extension naturelle en codimension supérieure de la condition $Y(q)$ est la notion de variété $\mathrm{CR} q$-concave. Les premiers résultats sur la résolution de l'équation de Cauchy-Riemann tangentielle dans les variétés CR $q$-concaves sont annoncés par Henkin dans [13], puis développés dans [1]. Dans sa thèse [3], le premier auteur a étendu les résultats de son article [4] au cas des variétés CR $q$-concaves, obtenant ainsi des noyaux de type Bochner-Martinelli avec des estimations $\mathcal{C}^{1 / 2-\varepsilon}$ sur $M$. Ces noyaux sont construits par itération de la résolution du $\bar{\partial}$ dans des domaines à coins $q$-convexe attachés à la variété $M$. Une "presque" formule d'homotopie (i.e., modulo un opérateur compact) avec estimations optimales est prouvée par Polyakov dans [19] pour les espaces de Stein et dans [20] pour les espaces $\mathcal{C}^{k}$. Dans ses articles Polyakov introduit la notion de variété régulièrement $q$-concave et obtient ses résultats sous cette hypothèse à priori plus restrictive que la $q$-concavité. Néanmoins il est prouvé dans [17] que si $M$ est $q$-concave, il existe $q^{\prime} \geq q$ tel que $M$ soit régulièrement $q^{\prime}$-concave et les résultats de Polyakov s'étendent donc au cas des variétés $q$-concaves.

Notre travail répond à une question de Henkin, posée au début des années 90, sur la possibilité de construire une solution fondamentale pour l'opérateur de CauchyRiemann tangentiel permettant d'obtenir un gain de régularité $1 / 2$, ce qui est optimal dans ce cadre.

L'article est organisé comme suit. Dans la Section 1, nous décrivons la situation géométrique et nous introduisons les principales notations. Dans la Section 2, nous montrons que l'existence d'une famille de noyaux satisfaisant une équation aux dérivées partielles adéquate et de bonnes conditions d'intégrabilité permet de prouver une formule de type Bochner-Martinelli-Koppelman dans $M$. La Section 3 est consacrée à montrer que les noyaux introduits dans [16] satisfont les propriétés exigées dans la Section 2 et permettent la construction d'une première solution fondamentale $B_{M}$ pour l'opérateur de Cauchy-Riemann tangentiel. Lorsque $M$ est une hypersurface, ces noyaux coïncident avec les noyaux définis par Fischer dans [9] et satisfont donc des estimations $\mathcal{C}^{l+1 / 2}$. En codimension $k>$ 1 , les noyaux utilisés ont une dépendance non linéaire par rapport à un paramètre $\lambda$ et nous sommes amenés à utiliser des résultats prouvés dans [16], rappelés ici en appendice (Section 6), pour montrer les conditions d'intégrabilité demandées. Par ailleurs les noyaux $B_{M}$ satisfont seulement des estimations $\mathcal{C}^{l+1 / 2-\varepsilon}, \varepsilon>0$. Nous utilisons ensuite, dans la Section 4 , une idée de [6] pour construire de nouveaux noyaux moins singuliers qui permettront d'obtenir les estimations optimales. La Section 5 est dévolue à l'étude de la continuité des opérateurs intégraux associés aux noyaux construits dans la Section 4. 
Cet article a été écrit pendant le séjour du second auteur à la Chalmers University à Göteborg puis à la Humboldt Universität à Berlin. Il souhaite remercier ses collègues Bo Berndtsson et Jürgen Leiterer pour leur accueil et les excellentes conditions de travail dont il a alors bénéficié.

\section{Situation Géométrique et Notations}

Soit $M$ une sous-variété différentiable de classe $\mathcal{C}^{2}$ de $\mathbb{C}^{n}$ de codimension réelle $k, 1 \leq k \leq n$, définie par

$$
M=\left\{z \in \omega \mid \hat{\rho}_{1}(z)=\cdots=\hat{\rho}_{k}(z)=0\right\},
$$

où $\omega$ est un ouvert de $\mathbb{C}^{n}$ et $\hat{\rho}_{1}, \ldots, \hat{\rho}_{k}$ des fonctions de classe $\mathcal{C}^{2}$ sur $\omega$ à valeurs réelles qui vérifient $d \hat{\rho}_{1}(z) \wedge \cdots \wedge d \hat{\rho}_{k}(z) \neq 0$ pour tout $z \in M$.

On note $T_{z}^{\mathbb{C}} M$ l'espace tangent complexe à $M$ au point $z \in M$. On a

$$
T_{z}^{\mathbb{C}} M=\left\{\xi \in \mathbb{C}^{n} \mid \sum_{j=1}^{n} \frac{\partial \hat{\rho}_{v}}{\partial z_{j}}(z) \xi_{j}=0, v=1, \ldots, k\right\} .
$$

On suppose que $M$ est Cauchy-Riemann (CR) générique, c'est-à-dire que

$$
\operatorname{dim}_{\mathbb{C}} T_{z}^{\mathbb{C}} M=n-k
$$

pour tout $z \in M$, ce qui équivaut à

$$
\bar{\partial} \hat{\rho}_{1}(z) \wedge \cdots \wedge \bar{\partial} \hat{\rho}_{k}(z) \neq 0
$$

pout tout $z \in M$.

On suppose également que $M$ n'est pas totalement réelle (i.e., $k<n$ ) et que $M$ est $q$-concave, $1 \leq q \leq \frac{n-k}{2}$, c'est-à-dire que pour tout $z \in M$ et tout $x \in \mathbb{R}^{k} \backslash\{0\}$ la forme hermitienne sur $T_{z}^{\mathbb{C}} M, \sum_{\alpha, \beta} \frac{\partial^{2} \hat{\rho}_{x}}{\partial z_{\alpha} \partial \bar{z}_{\beta}} \xi_{\alpha} \bar{\xi}_{\beta}$, où $\hat{\rho}_{x}=x_{1} \hat{\rho}_{1}+\cdots+x_{k} \hat{\rho}_{k}$, possède au moins $q$ valeurs propres strictement négatives.

Fixons $z_{0} \in M$ et $U \subset \subset \omega$ un voisinage de $z_{0}$ dans $\mathbb{C}^{n}$. Puisque $M$ est $q$ concave, d'après le Lemme 3.1.1 de [1], il existe une constante $C>0$ telle que, pour $j=1, \ldots, k$, les fonctions

$$
\begin{gathered}
\rho_{j}=\hat{\rho}_{j}+C \sum_{\nu=1}^{k} \hat{\rho}_{v}^{2} \text { et } \\
\rho_{-j}=-\hat{\rho}_{j}+C \sum_{\nu=1}^{k} \hat{\rho}_{v}^{2}
\end{gathered}
$$

possèdent la propriété suivante:

pour tout $I \in \mathcal{I}$ et tout $\lambda \in \Delta_{I}$ la forme de Levi de la fonction $\rho_{\lambda}=$ $\lambda_{i_{1}} \rho_{i_{1}}+\cdots+\lambda_{i_{|I|}} \rho_{i_{|I|}}$ admet au moins $q+k$ valeurs propres strictement positives sur $U$, 
où $\mathcal{I}$ désigne l'ensemble des parties $I \subset\{ \pm 1, \ldots, \pm k\}$ telles que $|i| \neq|j|$ pour tous $i, j \in I$ tels que $i \neq j,|I|$ le nombre d'éléments de $I$ et $\Delta_{I}$ le simplexe des suites $\left(\lambda_{j}\right)_{j \in \mathbb{Z}}$ des nombres réels $\lambda_{j} \in[0,1]$ tels que $\lambda_{j}=0$ si $j \notin I$ et $\sum \lambda_{j}=1$.

On note $\mathcal{I}(l), 1 \leq l \leq k$, l'ensemble de tous les $I \in \mathcal{I}$ tels que $|I|=l$. On ordonne $I \in \mathcal{I}$ par le module de ses éléments et on note $\mathcal{I}^{\prime}(l), 1 \leq l \leq k$, l'ensemble des multi-indices de longueur $l$ tels que si $I=\left(i_{1}, \ldots, i_{l}\right)$ alors $\left|i_{v}\right|=v$ pour $v=$ $1, \ldots, l$. Si $I \in \mathcal{I}$ et $v \in\{1, \ldots,|I|\}$, on pose $I(\hat{v})=I \backslash\left\{i_{v}\right\}$, où $i_{v}$ est le vième élément de $I$ après avoir ordonné $I$.

Finalement on pose

$$
\operatorname{sgn} I= \begin{cases}1 & \text { si le nombre d'éléments négatifs est pair, } \\ -1 & \text { si le nombre d'éléments négatifs est impair. }\end{cases}
$$

Soient $I=\left(i_{1}, \ldots, i_{l}\right)$ un multi-indice de $\mathcal{I}(l), 1 \leq l \leq k$, et $D$ un domaine relativement compact dans $U$. On définit

$$
\begin{aligned}
D_{I} & =\left\{\rho_{i_{1}}<0\right\} \cap \cdots \cap\left\{\rho_{i_{|I|}}<0\right\} \cap D, \\
D_{I}^{*} & =\left\{\rho_{i_{1}}>0\right\} \cap \cdots \cap\left\{\rho_{i_{|I|}}>0\right\} \cap D, \\
S_{I} & =\left\{\rho_{i_{1}}=0=\cdots=\rho_{i_{|I|}}=0\right\} \cap D, \\
S_{\{j\}}^{+} & =\bar{D}_{\{j\}} \text { pour } j= \pm 1, \ldots, \pm k, \\
S_{I}^{+} & =S_{I(\widehat{|I|})} \cap \bar{D}_{\left\{i_{|I|}\right\}} \quad \text { si } I \in \mathcal{I} \text { et }|I| \geq 2, \\
\tilde{S}_{I} & =S_{I} \cap\left\{\rho_{|I|+1}>0\right\} \cap\left\{\rho_{-(|I|+1)}>0\right\} \quad \text { si } 1 \leq|I| \leq k-1 .
\end{aligned}
$$

Notons que

$$
S_{I}=S_{I(|I|+1)}^{+} \cup S_{I(-(|I|+1))}^{+} \cup \tilde{S}_{I}
$$

et que $\tilde{S}_{I}=\emptyset \mathrm{si}|I|=k-1$. Ces variétés sont orientées comme suit: $D_{I}$ et $D_{I}^{*}$ comme $\mathbb{C}^{n}$ pour tout $I \in \mathcal{I}, S_{\{j\}}^{+}$comme $D_{\{j\}}$ pour $j= \pm 1, \ldots, \pm k, S_{I}$ comme $\partial S_{I}^{+}$ pour tout $I \in \mathcal{I}, S_{I}^{+}$comme $S_{I(\widehat{|I|})}$ pour tout $I \in \mathcal{I}$ si $|I| \geq 2$ et $M \cap D$ comme $S_{I}$ si $I=\{1, \ldots, k\}$.

On étend les définitions précédentes au cas où $|I|=0$, c'est-à-dire $I=\emptyset$, en posant $S_{\emptyset}=D$ et $\tilde{S}_{\emptyset}=S_{\emptyset} \cap\left\{\rho_{1}>0\right\} \cap\left\{\rho_{-1}>0\right\}$.

Si $f$ est une fonction définie sur un ouvert $\Omega$ de $M$, on définit la norme hölderienne d'ordre $\alpha, 0<\alpha<1$, de $f$ par

$$
\|f\|_{\alpha, \Omega}=\sup _{z \in \Omega}|f(z)|+\sup _{\substack{z, \zeta \in \Omega \\ z \neq \zeta}} \frac{|f(z)-f(\zeta)|}{|z-\zeta|^{\alpha}} .
$$

Si $M$ est de classe $\mathcal{C}^{l}$, on note $\mathcal{C}^{l+\alpha}(\Omega)$ l'espace de Fréchet des fonctions de classe $\mathcal{C}^{l}$ sur $\Omega$ dont toutes les dérivées tangentielles d'ordre $l$ sont localement hölderienne d'ordre $\alpha$. Pour tout compact $K$ de $\Omega$, le maximum de la borne supérieure sur $K$ des dérivées tangentielles d'ordre inférieur à $l$ et de la norme hölderienne d'ordre $\alpha$ des dérivées tangentielles d'ordre $l$ définit une semi-norme $\operatorname{sur} \mathcal{C}^{l+\alpha}(\Omega)$. 
Si $f$ est une $(n, r)$-forme différentielle sur $\Omega \subset \subset M$, elle s'écrit

$$
f=\sum_{J} f_{J} d z \wedge d \bar{z}_{J}
$$

avec $d z=d z_{1} \wedge \cdots \wedge d z_{n}$ et $d \bar{z}_{J}=d \bar{z}_{j_{1}} \wedge \cdots \wedge d \bar{z}_{j_{r}}$, car $M$ est plongée dans $\mathbb{C}^{n}$. La norme de $f$ est alors donnée par le maximum des normes des $f_{J}$.

\section{Formule de Bochner-Martinelli-Koppelman pour les Variétés $\mathbf{C R}$}

L'objet de cette section est de montrer qu'une famille de noyaux satisfaisant une équation aux dérivées partielles adéquate et possédant de bonnes propriétés d'intégrabilité permet d'obtenir une formule de Bochner-Martinelli-Koppelman dans les variétés $\mathrm{CR}$ de codimension quelconque.

Nous nous plaçons dans la situation géométrique de la Section 1.

Pour $I \in \mathcal{I}, 0 I$ désigne le multi-indice $\left(0, i_{1}, \ldots, i_{|I|}\right)$, où $I=\left(i_{1}, \ldots, i_{|I|}\right)$ est ordonné en module croissant.

On pose $C_{0}(z, \zeta)=B(z, \zeta)$, où $B(z, \zeta)$ désigne le noyau de Bochner-MartinelliKoppelman dans $\mathbb{C}^{n}$, ce qui correspond à un multi-indice $I$ de longueur nulle.

On suppose que l'on sait associer à chaque multi-indice ordonné $I \in \mathcal{I}(l), 1 \leq$ $l \leq k$, des formes différentielles $C_{0 I}(z, \zeta)$ de degré $2 n-|I|-1$ et $C_{I}(z, \zeta)$ de degré $2 n-|I|$, de classe $\mathcal{C}^{1}$ pour $z \in \bar{D}_{I}$ et $\zeta \in \bar{D}_{I}^{*}$ tels que $z \neq \zeta$, qui vérifient l'équation aux dérivées partielles

$$
\bar{\partial}_{z} C_{0 I}+\bar{\partial}_{\zeta} C_{0 I}=C_{0 \delta(I)}-C_{I},
$$

où $C_{0 \delta(I)}=\sum_{\nu=1}^{|I|}(-1)^{\nu+1} C_{0 I(\hat{v})}$.

On suppose également que les noyaux $C_{I}$ satisfont les conditions d'annulation suivantes:

$$
\begin{aligned}
{\left[C_{I}(z, \zeta)\right]_{p, r}=0 } & \text { si } 0 \leq p \leq n \text { et } n-k-q+1 \leq r \leq n-k, \\
\bar{\partial}_{z}\left[C_{I}(z, \zeta)\right]_{p, n-k-q}=0 & \text { si } 0 \leq p \leq n,
\end{aligned}
$$

où $\left[C_{I}(z, \zeta)\right]_{p, r}$ désigne la partie de bidegré $(p, r)$ en $z$ de $C_{I}$.

Pour alléger les écritures on pose $B_{I}=C_{0 I}$ pour tout $I \in \mathcal{I}(l), 1 \leq l \leq k$. L'équation (2.1) s'écrit alors

$$
\bar{\partial}_{z} B_{I}+\bar{\partial}_{\zeta} B_{I}=B_{\delta(I)}-C_{I} .
$$

On suppose finalement que pour tout $I \in \mathcal{I}(l), 1 \leq l \leq k$, les noyaux $B_{I}$ vérifient les conditions d'intégrabilité suivantes:

- pour toute forme différentielle $f$ de classe $\mathcal{C}^{1}$ sur $U$ à support dans $D$,

$$
\int_{\zeta \in S_{I}} f(\zeta) \wedge B_{I}(z, \zeta)
$$

définit une forme différentielle continue sur $\bar{D}_{I}$ et de classe $\mathcal{C}^{\infty}$ sur $D_{I}$; 
- soit $j \in\{ \pm 1, \ldots, \pm k\}$ tel que $I \cup\{j\} \in \mathcal{I}$, pour toute forme différentielle $f$ de classe $\mathcal{C}^{1}$ sur $U$ à support dans $D, \int_{\zeta \in S_{I \cup\{j\}}^{+}} f(\zeta) \wedge B_{I}(z, \zeta)$ définit une forme différentielle continue sur $\bar{D}_{I}$ et de classe $\mathcal{C}^{\infty}$ sur $D_{I}$;

- $\operatorname{pour} I=J$ ou $I=\delta(K)$ et $J=K(\widehat{|K|})$ avec $K \in \mathcal{I}(l+1), \int_{\zeta \in \tilde{S}_{J}} f_{\varepsilon}(\zeta) \wedge B_{I}(z, \zeta)$ tend vers 0 , quand $\varepsilon$ tend vers 0 , si $f_{\varepsilon}$ est une forme différentielle de classe $\mathcal{C}^{1}$ sur $U$ à support dans $D \cap\left\{\left|\rho_{1}\right|<\varepsilon\right\} \cap \cdots \cap\left\{\left|\rho_{k}\right|<\varepsilon\right\}$ telle que $\left\|\bar{\partial} f_{\varepsilon}\right\|=O(1 / \varepsilon)$ et $\int_{\zeta \in \tilde{S}_{J}} f_{\varepsilon}(\zeta) \wedge B_{I}(z, \zeta)=o(\varepsilon)$, si de plus $\bar{\partial} f_{\varepsilon}=0$.

DÉFINITION 2.1. On appellera famille de noyaux adaptés à la variété CR générique, $q$-concave $M$ de codimension réelle $k$ dans $\mathbb{C}^{n}$, toute famille de noyaux $B_{I}$ et $C_{I}, I \in \mathcal{I}(l), 1 \leq l \leq k$, possédant les propriétés précédentes.

Lemme 2.2. Soit $f$ une $(n, r)$-forme de classe $\mathcal{C}^{1}$ à support compact dans $D, 1 \leq$ $r \leq n-k$. Pour tout multi-indice ordonné $I \in \mathcal{I}(l), 1 \leq l \leq k$, et pour tout $n-k-q+1 \leq r \leq n-k$, on a au sens des courants sur $\bar{D}_{I}$

$$
\begin{aligned}
& \bar{\partial}_{z} \int_{\zeta \in S_{I}^{+}} f(\zeta) \wedge B_{\delta(I)}(z, \zeta)+(-1)^{|I|} \int_{\zeta \in S_{I}^{+}} \bar{\partial} f(\zeta) \wedge B_{\delta(I)}(z, \zeta) \\
& =(-1)^{r+n}\left(\bar{\partial}_{z} \int_{\zeta \in S_{I}} f(\zeta) \wedge B_{I}(z, \zeta)+(-1)^{|I|+1} \int_{\zeta \in S_{I}} \bar{\partial} f(\zeta) \wedge B_{I}(z, \zeta)\right) .
\end{aligned}
$$

Démonstration. Chacune des intégrales qui apparaissent dans la formule (2.5) étant continue sur $\bar{D}_{I}$ et de classe $\mathcal{C}^{\infty}$ sur $D_{I}$, il suffit de prouver (2.5) pour $z \in$ $D_{I}$. On applique la relation (2.4), puis la formule de Stokes, et on remarque que les termes en $C_{I}$ disparaissent car seuls interviennent les parties de bidegré $(n, r)$ en $z$ des différents éléments.

Rappelons que si $|I|=0$, on a posé $B_{I}=C_{0}=B$, où $B$ désigne le noyau de Bochner-Martinelli-Koppelman dans $\mathbb{C}^{n}$.

Lemme 2.3. Soit $f$ une $(n, r)$-forme de classe $\mathcal{C}^{1}$ à support compact dans $D, 1 \leq$ $r \leq n-k$. Pour tout $0 \leq l \leq k-1$, si $r$ vérifie $n-k-q+1 \leq r \leq n-k$, on a au sens des courants sur $M$

$$
\begin{aligned}
\sum_{|I|=l}\left(\bar{\partial}_{z} \int_{\zeta \in S_{I}} f(\zeta) \wedge\right. & \left.B_{I}(z, \zeta)+(-1)^{|I|+1} \int_{\zeta \in S_{I}} \bar{\partial} f(\zeta) \wedge B_{I}(z, \zeta)\right) \\
=(-1)^{l+r+n} \sum_{|I|=l+1} & \left(\bar{\partial}_{z} \int_{\zeta \in S_{I}} f(\zeta) \wedge B_{I}(z, \zeta)\right. \\
& \left.+(-1)^{|I|+1} \int_{\zeta \in S_{I}} \bar{\partial} f(\zeta) \wedge B_{I}(z, \zeta)\right)+(*)_{l}(f)(z),
\end{aligned}
$$

avec 
$(*)_{l}(f)(z)$

$$
\begin{aligned}
& =\sum_{|I|=l}\left(\bar{\partial}_{z} \int_{\zeta \in \tilde{S}_{I}} f(\zeta) \wedge B_{I}(z, \zeta)+(-1)^{l+1} \int_{\zeta \in \tilde{S}_{I}} \bar{\partial} f(\zeta) \wedge B_{I}(z, \zeta)\right) \\
& +(-1)^{l} \sum_{\nu=1}^{l}(-1)^{\nu+1} \sum_{J \in \mathcal{I}^{\prime}(l+1, \hat{\nu})}\left(\bar{\partial}_{z} \int_{\zeta \in \tilde{S}_{J}} f(\zeta) \wedge B_{J}(z, \zeta)\right. \\
& \left.+(-1)^{l+1} \int_{\zeta \in \tilde{S}_{J}} \bar{\partial} f(\zeta) \wedge B_{J}(z, \zeta)\right) \\
& +(-1)^{l} \sum_{\nu=1}^{l}(-1)^{\nu} \sum_{J \in \mathcal{I}^{\prime}(l+1, \hat{\nu})}(-1)^{r+n+1}\left(\bar{\partial}_{z} \int_{\zeta \in \tilde{S}_{J(|I|)}^{l+1}} f(\zeta) \wedge B_{\delta(J)}(z, \zeta)\right. \\
& \left.+(-1)^{l+1} \int_{\zeta \in \tilde{S}_{J((I \mid)}^{l+1}} \bar{\partial} f(\zeta) \wedge B_{\delta(J)}(z, \zeta)\right),
\end{aligned}
$$

où $\tilde{S}_{J(\widehat{|I|})}^{l+1}=\tilde{S}_{J(\widehat{|I|})} \cap\left\{\rho_{i_{l+1}}<0\right\}$ avec $J(\widehat{|I|})=\left(i_{1}, \ldots, i_{v-1}, i_{v+1}, \ldots, i_{l}\right)$, pour $J=I(\hat{v})$ et $I=\left(i_{1}, \ldots, i_{l+1}\right) \in \mathcal{I}^{\prime}(l+1)$, si $l \geq 1$ et

$$
(*)_{0}(f)(z)=\bar{\partial}_{z} \int_{\zeta \in \tilde{S}_{\emptyset}} f(\zeta) \wedge B(z, \zeta)+(-1)^{l+1} \int_{\zeta \in \tilde{S}_{\emptyset}} \bar{\partial} f(\zeta) \wedge B(z, \zeta) .
$$

Démonstration. On applique le Lemme 2.2 et la formule de Stokes en utilisant les propriétés géométriques suivantes:

- si $|I|=l$, on a $S_{I}=S_{I(l+1)}^{+} \cup S_{I(-(l+1))}^{+} \cup \tilde{S}_{I}$;

- $\partial \tilde{S}_{J(\widehat{|I|})} \cap\left\{\rho_{i_{|I|}}<0\right\}=S_{I \cup\{v\}}^{+} \cup S_{I \cup\{-v\}}^{+} \cup \tilde{S}_{I}$, si $I \in \mathcal{I}^{\prime}(l+1, \hat{v})$.

Soit $f$ une $(n, r)$-forme de classe $\mathcal{C}^{1}$ à support compact dans $D$. La formule de Bochner-Martinelli-Koppelman s'écrit

$$
f(z)=(-1)^{r+n}\left(\bar{\partial}_{z} \int_{\zeta \in D} f(\zeta) \wedge B(z, \zeta)-\int_{\zeta \in D} \bar{\partial} f(\zeta) \wedge B(z, \zeta)\right) .
$$

Le second membre de la formule de Bochner-Martinelli-Koppelman correspond au premier membre de la formule démontrée dans le Lemme 2.3 lorsque $l=0$.

En posant $B_{M}=\sum_{I \in \mathcal{I}^{\prime}(k)} \operatorname{sgn}(I) B_{I}$, en remarquant que le choix des orientations identifie $M$ avec $\operatorname{sgn}(I) S_{I}$ pour $I \in \mathcal{I}^{\prime}(k)$ et en appliquant $k$ fois le Lemme 2.3, on obtient la proposition suivante:

Proposition 2.4. Soit $f$ une $(n, r)$-forme de classe $\mathcal{C}^{1}$ à support compact dans $D, n-k-q+1 \leq r \leq n-k$, alors on a la formule suivante au sens des courants sur $M$ 
$f(z)$

$$
\begin{aligned}
=(-1)^{(k+1)(r+n)+\frac{k(k-1)}{2}}\left(\bar{\partial}_{z} \int_{\zeta \in M} f(\zeta) \wedge B_{M}(z, \zeta)\right. \\
\left.\quad+(-1)^{k+1} \int_{\zeta \in M} \bar{\partial} f(\zeta) \wedge B_{M}(z, \zeta)\right)+\sum_{l=0}^{k-1}(*)_{l}(f)(z)
\end{aligned}
$$

THÉORÈme 2.5. Soit $f$ une $(n, r)$-forme de classe $\mathcal{C}^{1}$ à support compact dans $D \cap M, n-k-q+1 \leq r \leq n-k$, alors on a la formule suivante au sens des courants sur $M$

$$
\begin{aligned}
f(z)=(-1)^{(k+1)(r+n)+\frac{k(k-1)}{2}}( & \bar{\partial}_{b} \int_{\zeta \in M} f(\zeta) \wedge B_{M}(z, \zeta) \\
& \left.+(-1)^{k+1} \int_{\zeta \in M} \bar{\partial}_{b} f(\zeta) \wedge B_{M}(z, \zeta)\right)
\end{aligned}
$$

Démonstration. Soit $\tilde{f}$ une extension de classe $\mathcal{C}^{1}$ de $f$ à un voisinage de $M$. On considère une fonction $\chi \in \mathcal{C}^{\infty}(\mathbb{R})$ telle que $\chi=1$ sur $(-\infty, 1 / 2]$ et $\chi=0$ sur $[1,+\infty)$. On pose $\chi_{\varepsilon}(z)=\prod_{j= \pm 1, \ldots, \pm k} \chi\left(\rho_{j}(z) / \varepsilon\right)$ pour $z \in U$ et $\varepsilon>0$; il existe alors une constante $A>0$ telle que $\left\|d \chi_{\varepsilon}(z)\right\| \leq A / \varepsilon$ pour $z \in U$ et $\varepsilon>0$. On pose $\tilde{f}_{\varepsilon}=\gamma_{\varepsilon} \tilde{f}$. Il résulte alors de la Proposition 2.4 et des propriétés d'intégrabilité des noyaux $B_{I}$ que

$$
\begin{aligned}
\lim _{\varepsilon \rightarrow 0} \tilde{f}_{\varepsilon}(z)=(-1)^{(k+1)(r+n)+\frac{k(k-1)}{2}} \lim _{\varepsilon \rightarrow 0}( & \bar{\partial}_{z} \int_{\zeta \in M} \tilde{f}_{\varepsilon}(\zeta) \wedge B_{M}(z, \zeta) \\
& \left.+(-1)^{k+1} \int_{\zeta \in M} \bar{\partial} \tilde{f}_{\varepsilon}(\zeta) \wedge B_{M}(z, \zeta)\right) .
\end{aligned}
$$

De plus, par définition de $\tilde{f}_{\varepsilon}$, on a, pour tout $\varepsilon>0$,

$$
\left.\tilde{f}_{\varepsilon}\right|_{M}=f
$$

ce qui termine la démonstration du théorème.

Pour obtenir une formule de Bochner-Martinelli-Koppelman dans $M$, nous devons préciser quelques propriétés du noyau $B_{M}$. Pour alléger les notations, nous noterons encore $\bar{\partial}_{z, \zeta}=\bar{\partial}_{z}+\bar{\partial}_{\zeta}$ l'équation de Cauchy-Riemann tangentielle sur $M \times M$.

LEMME 2.6. Le noyau $B_{M}$ est une forme différentielle de classe $\mathcal{C}^{1}$ sur

$$
(M \cap U) \times(M \cap U) \backslash\{(z, \zeta) \in(M \cap U) \times(M \cap U) \mid z=\zeta\},
$$

de degré $2 n-k-1$, tel que pour $0 \leq p \leq n$ et $n-k-q+1 \leq r \leq n-k$

$$
\bar{\partial}_{\zeta}\left[B_{M}\right]_{p, r}=-\bar{\partial}_{z}\left[B_{M}\right]_{p, r-1},
$$

où $\left[B_{M}\right]_{p, r}$ désigne la composante de bidegré $(p, r)$ en $z$ de $B_{M}$ (on pose $\left.\left[B_{M}\right]_{p, n-k}=0\right)$. 
Démonstration. Rappelons que $B_{M}=\sum_{I \in \mathcal{I}^{\prime}(k)} \operatorname{sgn}(I) B_{I}$. La formule (2.4) donne alors immédiatement

$$
\bar{\partial}_{z} B_{M}+\bar{\partial}_{\zeta} B_{M}=\sum_{I \in \mathcal{I}^{\prime}(k)} \operatorname{sgn}(I) B_{\delta(I)}-\sum_{I \in \mathcal{I}^{\prime}(k)} \operatorname{sgn}(I) C_{I} .
$$

On sait, grâce aux propriétés d'annulation des noyaux $C_{I}$ que $\left[C_{I}(z, \zeta)\right]_{p, r}=0$ si $0 \leq p \leq n$ et $n-k-q+1 \leq r \leq n-k$, par conséquent

$$
\bar{\partial}_{z}\left[B_{M}\right]_{p, r-1}+\bar{\partial}_{\zeta}\left[B_{M}\right]_{p, r}=\sum_{I \in \mathcal{I}^{\prime}(k)} \operatorname{sgn}(I)\left[B_{\delta(I)}\right]_{p, r} .
$$

De plus par définition de $\operatorname{sgn}(I)$ et $\delta(I)$, on a $\sum_{I \in \mathcal{I}^{\prime}(k)} \operatorname{sgn}(I)\left[B_{\delta(I)}\right]_{p, r}=0$. En effet pour un multi-indice donné $J \in \mathcal{I}(k-1)$ et pour un entier $v$ tel que $\pm v \notin$ $J$, il existe deux multi-indices $I_{1}$ et $I_{2}$ dans $\mathcal{I}(k)$ tels que $J=I_{1}(\hat{v})=I_{2}(\hat{v})$ qui vérifient $\operatorname{sgn}\left(I_{1}\right)=-\operatorname{sgn}\left(I_{2}\right)$ car, d'un point de vue ensembliste, si $I_{1}=J \cup\{v\}$ alors $I_{2}=J \cup\{-v\}$.

THÉORÈME 2.7. Soit $\Omega$ un domaine à bord $\mathcal{C}^{1}$ relativement compact dans $M \cap U$ et $f$ une $(n, r)$-forme de classe $\mathcal{C}^{1}$ dans $\bar{\Omega}, n-k-q+1 \leq r \leq n-k$, alors on a la formule suivante au sens des courants sur $M$

$$
\begin{aligned}
(-1)^{(k+1)(r+n)+\frac{k(k-1)}{2}} f(z)= & \bar{\partial}_{b} \int_{\zeta \in \Omega} f(\zeta) \wedge B_{M}(z, \zeta) \\
& +(-1)^{k+1} \int_{\zeta \in \Omega} \bar{\partial}_{b} f(\zeta) \wedge B_{M}(z, \zeta) \\
& +(-1)^{k} \int_{\zeta \in \partial \Omega} f(\zeta) \wedge B_{M}(z, \zeta)
\end{aligned}
$$

Démonstration. Soit $z \in \Omega$ fixé et $\chi$ une fonction de classe $\mathcal{C}^{2}$ à support compact dans $\Omega$ telle que $\chi \equiv 1$ dans un voisinage de $z$. D'après le Lemme 2.6, comme $f$ est de bidegré $(n, r)$ avec $n-k-q+1 \leq r \leq n-k$, on a

$$
\begin{aligned}
d_{\zeta}\left((1-\chi(\zeta)) f(\zeta) \wedge\left[B_{M}\right]_{n, r}(z, \zeta)\right)= & \bar{\partial}_{b} f(\zeta) \wedge\left[B_{M}\right]_{n, r}(z, \zeta) \\
& -\bar{\partial}_{b}(\chi(\zeta) f(\zeta)) \wedge\left[B_{M}\right]_{n, r}(z, \zeta) \\
& -\bar{\partial}_{b, z}\left((1-\chi(\zeta)) f(\zeta) \wedge\left[B_{M}\right]_{n, r-1}(z, \zeta)\right)
\end{aligned}
$$

Puisque la fonction $\chi$ est à support dans $\Omega$, donc nulle sur $\partial \Omega$, on obtient par la formule de Stokes

$$
\begin{aligned}
\int_{\zeta \in \partial \Omega} f(\zeta) \wedge\left[B_{M}\right]_{n, r}(z, \zeta) & =\int_{\zeta \in \partial \Omega}(1-\chi(\zeta)) f(\zeta) \wedge\left[B_{M}\right]_{n, r}(z, \zeta) \\
& =\int_{\zeta \in \Omega} d\left((1-\chi(\zeta)) f(\zeta) \wedge\left[B_{M}\right]_{n, r}(z, \zeta)\right)
\end{aligned}
$$

et par conséquent 


$$
\begin{aligned}
\int_{\zeta \in \partial \Omega} f(\zeta) \wedge\left[B_{M}\right]_{n, r}(z, \zeta)= & \int_{\zeta \in \Omega} \bar{\partial}_{b} f(\zeta) \wedge\left[B_{M}\right]_{n, r}(z, \zeta) \\
& +(-1)^{k+1}\left(\bar{\partial}_{b, z} \int_{\zeta \in \Omega} f(\zeta) \wedge\left[B_{M}\right]_{n, r-1}(z, \zeta)\right) \\
& -\int_{\zeta \in \Omega} \bar{\partial}_{b}(\chi(\zeta) f(\zeta)) \wedge\left[B_{M}\right]_{n, r}(z, \zeta) \\
& +(-1)^{k}\left(\bar{\partial}_{b, z} \int_{\zeta \in \Omega} \chi(\zeta) f(\zeta) \wedge\left[B_{M}\right]_{n, r-1}(z, \zeta)\right)
\end{aligned}
$$

On applique alors le Théorème 2.5 à $\chi f$ et la formule est démontrée.

\section{Construction de Noyaux Adaptés à une Variété CR}

Etant donnée une sous-variété $\mathrm{CR}$ générique, $q$-concave $M$ de classe $\mathcal{C}^{3}$, de codimension réelle $k$ dans $\mathbb{C}^{n}$ et un point $z_{0}$ de $M$, nous allons construire une famille de noyaux adaptés à $M$ au sens de la Définition 2.1 au voisinage de $z_{0}$.

Nous reprenons les notations de la Section 1.

DÉfINITION 3.1. Une section de Leray associée à $M$ au voisinage de $\bar{D}$ est une application $\psi$ qui associe à chaque multi-indice $I \in \mathcal{I}(l), 1 \leq l \leq k$, une application à valeurs dans $\mathbb{C}^{n}$

$$
\psi_{I}(z, \zeta, \lambda)=\left(\psi_{I}^{1}(z, \zeta, \lambda), \ldots, \psi_{I}^{n}(z, \zeta, \lambda)\right)
$$

définie et de classe $\mathcal{C}^{2}$ pour $z \in \bar{D}_{I}, \zeta \in \bar{D}_{I}^{*}$ tels que $z \neq \zeta$ et $\lambda \in \Delta_{I}$, telle que

$$
\left\langle\psi_{I}(z, \zeta, \lambda), \zeta-z\right\rangle=1
$$

et

$$
\left.\psi_{I}\right|_{\left(\bar{D}_{J} \times \bar{D}_{J}^{*}\right) \backslash\{z=\zeta\} \times \Delta_{I}}=\left.\psi_{J}\right|_{\left(\bar{D}_{J} \times \bar{D}_{J}^{*}\right) \backslash\{z=\zeta\} \times \Delta_{I}} \quad \text { si } I \subset J .
$$

On note $\stackrel{\circ}{\chi}$ une fonction de classe $\mathcal{C}^{\infty}$ de $[0,1]$ dans lui-même qui vérifie $\stackrel{\circ}{\chi}(\lambda)=0$ si $0 \leq \lambda \leq 1 / 4$ et $\stackrel{\circ}{\chi}(\lambda)=1$ si $1 / 2 \leq \lambda \leq 1$.

Si $I \in \mathcal{I}(l), 1 \leq l \leq k$, pour $\lambda \in \Delta_{0 I}$ tel que $\lambda_{0} \neq 1$, on note $\lambda$ le point de $\Delta_{I}$ défini par

$$
\stackrel{\circ}{\lambda}_{i_{v}}=\frac{\lambda_{i_{v}}}{1-\lambda_{0}} \quad(v=1, \ldots, l) .
$$

Soit $\psi$ une section de Leray associée à $M$ au voisinage de $\bar{D}$, on pose

$$
\psi_{0 I}(z, \zeta, \lambda)=\stackrel{\circ}{\chi}\left(\lambda_{0}\right) \frac{\bar{\zeta}-\bar{z}}{|\zeta-z|^{2}}+\left(1-\stackrel{\circ}{\chi}\left(\lambda_{0}\right)\right) \psi_{I}(z, \zeta, \stackrel{\circ}{\lambda})
$$

pour tout $I \in \mathcal{I}(l), 1 \leq l \leq k, z \in \bar{D}_{I}, \zeta \in \bar{D}_{I}^{*}$ tels que $z \neq \zeta$ et $\lambda \in \Delta_{0 I}$. Notons que $\psi_{0 I}$ est aussi de classe $\mathcal{C}^{2}$.

On peut alors définir les noyaux $K_{0 I}(z, \zeta, \lambda)$, pour $z \in \bar{D}_{I}, \zeta \in \bar{D}_{I}^{*}$ tels que $z \neq$ $\zeta$ et $\lambda \in \Delta_{0 I}$, par 
$K_{0 I}(z, \zeta, \lambda)=\frac{(-1)^{n(n-1) / 2}}{(2 i \pi)^{n}}\left\langle\psi_{0 I}, d(\zeta-z)\right\rangle \wedge\left\langle\left(\bar{\partial}_{z, \zeta}+d_{\lambda}\right) \psi_{0 I}, d(\zeta-z)\right\rangle^{n-1}$,

et les noyaux $K_{I}(z, \zeta, \lambda)$ par

$K_{I}(z, \zeta, \lambda)=\frac{(-1)^{n(n-1) / 2}}{(2 i \pi)^{n}}\left\langle\psi_{I}, d(\zeta-z)\right\rangle \wedge\left\langle\left(\bar{\partial}_{z, \zeta}+d_{\lambda}\right) \psi_{I}, d(\zeta-z)\right\rangle^{n-1}$.

Les noyaux $K_{0 I}$ et $K_{I}$ sont des formes différentielles de classe $\mathcal{C}^{1}$ et de degré $2 n-1$ et on a

$$
\left(\bar{\partial}_{z, \zeta}+d_{\lambda}\right) K_{0 I}(z, \zeta, \lambda)=0
$$

d'après la Proposition 3.9 de [14].

Pour finir on pose, pour $z \in \bar{D}_{I}, \zeta \in \bar{D}_{I}^{*}$ tels que $z \neq \zeta$,

$$
\begin{gathered}
C_{0 I}(z, \zeta)=\int_{\lambda \in \Delta_{0 I}} K_{0 I}(z, \zeta, \lambda), \\
C_{I}(z, \zeta)=\int_{\lambda \in \Delta_{I}} K_{I}(z, \zeta, \lambda) .
\end{gathered}
$$

Proposition 3.2. Les noyaux $C_{0 I}(z, \zeta)$ et $C_{I}(z, \zeta)$ sont des formes différentielles respectivement de degré $2 n-|I|-1$ et de degré $2 n-|I|$, de classe $\mathcal{C}^{1}$ pour $z \in$ $\bar{D}_{I}$ et $\zeta \in \bar{D}_{I}^{*}$ tels que $z \neq \zeta$, qui vérifient l'équation aux dérivées partielles

$$
\bar{\partial}_{z} C_{0 I}+\bar{\partial}_{\zeta} C_{0 I}=C_{0 \delta(I)}-C_{I},
$$

où $C_{0 \delta(I)}=\sum_{v=1}^{|I|}(-1)^{v+1} C_{0 I(\hat{v})}$.

Démonstration. En appliquant la formule de Stokes on a

$$
\int_{\lambda \in \partial \Delta_{0 I}} K_{0 I}(z, \zeta, \lambda)=\int_{\lambda \in \Delta_{0 I}} d_{\lambda} K_{0 I}(z, \zeta, \lambda)
$$

Mais $\partial \Delta_{0 I}=\sum_{\nu=1}^{|I|}(-1)^{\nu} \Delta_{0 I(\hat{v})}+\Delta_{I}$, on a donc par définition des noyaux $C_{0 I}$, $C_{I}$, et $C_{0 \delta(I)}$,

$$
\int_{\lambda \in \partial \Delta_{0 I}} K_{0 I}(z, \zeta, \lambda)=-C_{0 \delta(I)}+C_{I} .
$$

Comme $\left(\bar{\partial}_{z, \zeta}+d_{\lambda}\right) K_{0 I}=0$, on obtient

$$
\int_{\lambda \in \Delta_{0 I}} d_{\lambda} K_{0 I}(z, \zeta, \lambda)=-\int_{\lambda \in \Delta_{0 I}} \bar{\partial}_{z, \zeta} K_{0 I}(z, \zeta, \lambda)=-\left(\bar{\partial}_{z} C_{0 I}+\bar{\partial}_{\zeta} C_{0 I}\right),
$$

d'où le résultat.

\subsection{Conditions d'Annulation}

Les propriétés d'annulation des noyaux $C_{I}$ sont liées à des propriétés d'holomorphie de la section de Leray $\psi$ associée à $M$ à partir de laquelle ils sont construits.

DÉFINITION 3.3. Une application $f$ définie sur une variété analytique complexe $X$ à valeurs dans $\mathbb{C}^{n}$ est dite $m$-holomorphe, si pour tout point $\xi \in X$, il existe 
des coordonnées holomorphes $h_{1}, \ldots, h_{n}$ dans un voisinage de $\xi$ telles que $f$ soit holomorphe par rapport à $h_{1}, \ldots, h_{m}$.

Nous dirons que la section de Leray $\psi$ associée à $M$ est $m$-holomorphe par rapport à la variable $z$, si pour tout $I \in \mathcal{I}(l), 1 \leq l \leq k$, chacune des applications $\psi_{I}$ est $m$-holomorphe par rapport à la variable $z$.

On prouve alors facilement le lemme suivant:

LEMME 3.4. On suppose que la section de Leray $\psi$ associée à $M$ au voisinage $d u$ domaine $\bar{D}$ est $(q+k)$-holomorphe par rapport à la variable $z$. Soit $I \in \mathcal{I}(l)$, $1 \leq l \leq k ;$ si $\left[C_{I}(z, \zeta)\right]_{p, r}$ désigne la partie de bidegré $(p, r)$ en $z$ de $C_{I}$, alors pour tout $\zeta \in \bar{D}_{I}^{*}$ fixé

$$
\begin{aligned}
{\left[C_{I}(z, \zeta)\right]_{p, r}=0 } & \text { si } 0 \leq p \leq n \text { et } n-k-q+1 \leq r \leq n-k, \\
\bar{\partial}_{z}\left[C_{I}(z, \zeta)\right]_{p, n-k-q}=0 & \text { si } 0 \leq p \leq n
\end{aligned}
$$

$\operatorname{sur} \bar{D}_{I} \backslash\{\zeta\}$.

On se place dans la situation géométrique décrite dans la Section 1 et on note $G(n, p)$ la grassmannienne des sous espaces vectoriels de dimension $p$ de $\mathbb{C}^{n}$. La variété $M$ étant $q$-concave, d'après [17], si $q^{\prime} \geq q$ est l'ordre de concavité maximale de $M$ en $z_{0}$, c'est-à-dire $M$ est $q^{\prime}$-concave en $z_{0}$ mais n'est pas $\left(q^{\prime}+1\right)$ concave en $z_{0}$, on peut choisir $D$ assez petit pour qu'il existe une application de classe $\mathcal{C}^{\infty}$

$$
T: \mathbb{R}^{k} \backslash\{0\} \rightarrow G\left(n, q^{\prime}+k\right)
$$

telle que la forme de Levi au voisinage de $\bar{D}$ de la fonction

$$
\tilde{\rho}_{x}=\sum_{i=1}^{k} x_{i} \hat{\rho}_{i}+C\left(\sum_{i=1}^{k}\left|x_{i}\right|\right)\left(\sum_{\nu=1}^{k} \hat{\rho}_{v}^{2}\right)
$$

soit définie positive sur $T(x)$ pour tout $x \in \mathbb{R}^{k} \backslash\{0\}$.

Soit $\left(e_{1}, \ldots, e_{k}\right)$ la base canonique de $\mathbb{R}^{k}$, on pose $e_{-j}=-e_{j}$ pour tout $1 \leq j \leq$ $k$. Si $I=\left(i_{1}, \ldots, i_{l}\right) \in \mathcal{I}(l), 1 \leq l \leq k$, on définit le simplexe géométrique $\tilde{\tilde{\Delta}}_{I}$ par

$$
\tilde{\Delta}_{I}=\left\{x \in \mathbb{R}^{k} \mid x=\sum_{j=1}^{l} \lambda_{i_{j}} e_{i_{j}}, \lambda_{i_{j}} \geq 0,1 \leq j \leq l, \sum_{j=1}^{l} \lambda_{i_{j}}=1\right\} .
$$

On identifie alors le simplex abstrait $\Delta_{I}$ et le simplexe géométrique $\tilde{\Delta}_{I}$ en posant $x(\lambda)=\sum_{j=1}^{l} \lambda_{i_{j}} e_{i_{j}}$ pour tout $\lambda \in \Delta_{I}$. On remarque qu'alors $\tilde{\rho}_{x(\lambda)}=\rho_{\lambda}$.

En restreignant $T$ à $\tilde{\Delta}_{I}$ et après l'identification de $\Delta_{I}$ avec $\tilde{\Delta}_{I}$ on obtient une application

$$
T_{I}: \Delta_{I} \rightarrow G\left(n, q^{\prime}+k\right)
$$

de classe $\mathcal{C}^{\infty}$ telle que la forme de Levi au voisinage de $\bar{D}$ de la fonction $\rho_{\lambda}$ soit définie positive sur $T_{I}(\lambda)$ pour tout $\lambda \in \Delta_{I}$. Si $I$ et $J$ sont deux éléments de $\mathcal{I}$ tels que $I \subset J$ on a alors $\left.T_{J}\right|_{\Delta_{I}}=T_{I}$. 
Comme dans [16], on peut alors construire pour les domaines $D_{I}$ des sections de Leray possédant de bonnes propriétés d'holomorphie. Si $M$ est de classe $\mathcal{C}^{3}$, ces sections seront de classe $\mathcal{C}^{2}$. Rappelons les principales étapes de cette construction.

Pour $\lambda \in \Delta_{I}$, on note $F_{\lambda}(\cdot, \zeta)$ le polynôme de Levi de $\rho_{\lambda}$ au point $\zeta \in U$. Pour $\zeta \in U, z \in \mathbb{C}^{n}$

$$
F_{\lambda}(z, \zeta)=2 \sum_{j=1}^{n} \frac{\partial \rho_{\lambda}}{\partial \zeta_{j}}(\zeta)\left(\zeta_{j}-z_{j}\right)-\sum_{j, k=1}^{n} \frac{\partial^{2} \rho_{\lambda}}{\partial \zeta_{j} \partial \zeta_{k}}(\zeta)\left(\zeta_{j}-z_{j}\right)\left(\zeta_{k}-z_{k}\right)
$$

On note $P^{\lambda}$ la projection orthogonale de $\mathbb{C}^{n}$ sur $T_{I}(\lambda)$ et on pose $Q^{\lambda}=I_{\mathbb{C}^{n}}-P^{\lambda}$, où $I_{\mathbb{C}^{n}}$ désigne l'application identique de $\mathbb{C}^{n}$. La formule de Taylor implique que, si le domaine $D \subset \subset U$ est assez petit, il existe des constantes strictement positives $\alpha$ et $A$ telles que

$$
\operatorname{Re} F_{\lambda}(z, \zeta) \geq \rho_{\lambda}(\zeta)-\rho_{\lambda}(z)+\alpha|\zeta-z|^{2}-A\left|Q^{\lambda}(\zeta-z)\right|^{2}
$$

pour $\zeta, z$ au voisinage de $\bar{D}$.

Puisque $\rho_{\lambda}$ est de classe au moins $\mathcal{C}^{2}$ dans $\omega$, on peut trouver des fonctions $a_{j k}^{\lambda}$, $j, k=1, \ldots, n$, de classe $\mathcal{C}^{\infty}$ sur $U$, dépendant linéairement de $\lambda$, telles que pour tout $\zeta \in U$

$$
\left|a_{j k}^{\lambda}(\zeta)-\frac{\partial^{2} \rho_{\lambda}}{\partial \zeta_{j} \partial \zeta_{k}}(\zeta)\right|<\frac{\alpha}{2 n^{2}} .
$$

Alors en posant

$$
\tilde{F}_{\lambda}(z, \zeta)=2 \sum_{j=1}^{n} \frac{\partial \rho_{\lambda}}{\partial \zeta_{j}}(\zeta)\left(\zeta_{j}-z_{j}\right)-\sum_{j, k=1}^{n} a_{j k}^{\lambda}(\zeta)\left(\zeta_{j}-z_{j}\right)\left(\zeta_{k}-z_{k}\right),
$$

on déduit de (3.5) et (3.6) que

$$
\operatorname{Re} \tilde{F}_{\lambda}(z, \zeta) \geq \rho_{\lambda}(\zeta)-\rho_{\lambda}(z)+\frac{\alpha}{2}|\zeta-z|^{2}-A\left|Q^{\lambda}(\zeta-z)\right|^{2}
$$

pour $\zeta, z$ au voisinage de $\bar{D}$.

On note $\left(Q_{j k}^{\lambda}\right)_{j, k=1}^{n}$ les coefficients de la matrice $Q^{\lambda}$, et, pour $(z, \zeta, \lambda) \in$ $\mathbb{C}^{n} \times U \times \Delta_{I}$, on pose

$$
\begin{aligned}
w_{j}(z, \zeta, \lambda) & =2 \frac{\partial \rho_{\lambda}}{\partial \zeta_{j}}(\zeta)-\sum_{k=1}^{n} a_{j k}^{\lambda}(\zeta)\left(\zeta_{k}-z_{k}\right)+A \sum_{k=1}^{n} \overline{Q_{j k}^{\lambda}\left(\zeta_{k}-z_{k}\right)} \\
w(z, \zeta, \lambda) & =\left(w_{1}(z, \zeta, \lambda), \ldots, w_{n}(z, \zeta, \lambda)\right) \\
\Phi(z, \zeta, \lambda) & =\langle w(z, \zeta, \lambda), \zeta-z\rangle
\end{aligned}
$$

Comme $Q^{\lambda}$ est une projection orthogonale, on a

$$
\Phi(z, \zeta, \lambda)=\tilde{F}_{\lambda}(z, \zeta)+A\left|Q^{\lambda}(\zeta-z)\right|^{2}
$$

et on déduit de (3.7) que

$$
\operatorname{Re} \Phi(z, \zeta, \lambda) \geq \rho_{\lambda}(\zeta)-\rho_{\lambda}(z)+\frac{\alpha}{2}|\zeta-z|^{2}
$$

pour $\zeta, z$ au voisinage de $\bar{D}$ et $\lambda \in \Delta_{I}$. 
Remarquons que, pour $\zeta$ fixé dans un voisinage de $\bar{D}$ et $\lambda \in \Delta_{I}$, on peut choisir de nouvelles coordonnées $h_{1}, \ldots, h_{n}$ dépendant linéairement des coordonnées complexes initiales telles que

$$
\left\{z \in \mathbb{C}^{n} \mid Q^{\lambda}(z)=0\right\}=\left\{z \in \mathbb{C}^{n} \mid h_{q^{\prime}+k+1}(z)=\cdots=h_{n}(z)=0\right\} .
$$

L'application $z \mapsto \overline{Q^{\lambda}(\zeta-z)}$ est alors indépendante de $h_{1}, \ldots, h_{q^{\prime}+k}$, ce qui implique que $w(\cdot, \zeta, \lambda)$ est une application $\mathbb{C}$-linéaire par rapport à $h_{1}, \ldots, h_{q^{\prime}+k}$ et que $\Phi(\cdot, \zeta, \lambda)$ est un polynôme quadratique complexe en $h_{1}, \ldots, h_{q^{\prime}+k}$.

Par conséquent en posant

$$
\psi_{I}(z, \zeta, \lambda)=\frac{w(z, \zeta, \lambda)}{\Phi(z, \zeta, \lambda)}
$$

pour $(z, \zeta, \lambda) \in \bar{D}_{I} \times \bar{D}_{I}^{*} \times \Delta_{I}, I \in \mathcal{I}(l), 1 \leq l \leq k$, on obtient une section de Leray $\psi=\left(\psi_{I}\right)_{I \in \bigcup_{1 \leq l \leq k} \mathcal{I}(l)}$ associée à $M$ au voisinage du domaine $\bar{D}$, qui est $\left(q^{\prime}+k\right)$-holomorphe et donc $(q+k)$-holomorphe, puisque $q^{\prime} \geq q$, par rapport à la variable $z$.

\subsection{Conditions d'Intégrabilité}

Dans un premier temps nous allons prouver que les noyaux $B_{I}=C_{0 I}, I \in \mathcal{I}(l)$, $1 \leq l \leq k$, construits à l'aide de la section de Leray $\psi=\left(\psi_{I}\right)_{I \in \cup_{1 \leq l \leq k} \mathcal{I}(l)}$ associée à $M$ au voisinage du domaine $\bar{D}$, définie dans le paragraphe précédent, vérifient les conditions d'intégrabilité:

- pour toute forme différentielle $f$ de classe $\mathcal{C}^{1}$ sur $U$ à support dans $D$,

$$
\int_{\zeta \in S_{I}} f(\zeta) \wedge B_{I}(z, \zeta)
$$

définit une forme différentielle continue sur $\bar{D}_{I}$ et de classe $\mathcal{C}^{\infty}$ sur $D_{I}$ :

- soit $j \in\{ \pm 1, \ldots, \pm k\}$ tel que $I \cup\{j\} \in \mathcal{I}$, pour toute forme différentielle $f$ de classe $\mathcal{C}^{1}$ sur $U$ à support dans $D, \int_{\zeta \in S_{I \cup\{j\}}^{+}} f(\zeta) \wedge B_{I}(z, \zeta)$ définit une forme différentielle continue sur $\bar{D}_{I}$ et de classe $\mathcal{C}^{\infty}$ sur $D_{I}$.

Nous devons donc considérer les formes différentielles

$$
\begin{aligned}
\int_{\zeta \in S_{I}} f(\zeta) \wedge B_{I}(z, \zeta) & =\int_{(\zeta, \lambda) \in S_{I} \times \Delta_{0 I}} f(\zeta) \wedge K_{0 I}(z, \zeta, \lambda), \\
\int_{\zeta \in S_{I \cup\{j\}}^{+}} f(\zeta) \wedge B_{I}(z, \zeta) & =\int_{(\zeta, \lambda) \in S_{I \cup\{j\}}^{+} \times \Delta_{0 I}} f(\zeta) \wedge K_{0 I}(z, \zeta, \lambda) .
\end{aligned}
$$

Remarquons que les formes $\psi_{I}$ et (respectivement $\psi_{0 I}$ ) sont définies pour $z \in \bar{D}_{I}$, $\zeta \in \bar{D}_{I}^{*}$ tels que $z \neq \zeta$ et $\lambda \in \Delta_{I}$ (respectivement $\lambda \in \Delta_{0 I}$ ) et de classe $\mathcal{C}^{2}$ en $\zeta$ et $\mathcal{C}^{\infty}$ en $z$ sur leur domaine de définition. Par conséquent les noyaux $B_{I}$ sont de classe $\mathcal{C}^{1}$ en $\zeta$ et $\mathcal{C}^{\infty}$ en $z$ pour $(z, \zeta) \in D_{I} \times \bar{D}_{I}^{*}$. Les formes différentielles (3.10) sont donc définies et de classe $\mathcal{C}^{\infty}$ sur $D_{I}$. Nous allons prouver qu'elles se prolongent en des formes différentielles continues sur $\bar{D}_{I}$. 
On considère pour $I=\left(i_{1}, \ldots, i_{l}\right) \in \mathcal{I}(l)$ les sous-variétés $\Gamma_{I}=\left\{\zeta \in \bar{D}_{I}^{*} \mid\right.$ $\left.\rho_{i_{1}}=\cdots=\rho_{i_{l}}\right\}$ et on les oriente de telle sorte que l'orientation de $\partial \Gamma_{I}$ coïncide avec celle de $S_{I}$.

Si $f$ est une $(n, r)$-forme différentielle de classe $\mathcal{C}^{1}$ sur $U$ à support dans $D$, on a alors pour $z \in D_{I}$

$$
\begin{aligned}
\int_{\zeta \in S_{I}} f(\zeta) \wedge B_{I}(z, \zeta)= & \int_{(\zeta, \lambda) \in S_{I} \times \Delta_{0 I}} f(\zeta) \wedge K_{0 I}(z, \zeta, \lambda) \\
= & \int_{(\zeta, \lambda) \in \Gamma_{I} \times \Delta_{0 I}} \bar{\partial} f(\zeta) \wedge K_{0 I}(z, \zeta, \lambda) \\
& +(-1)^{n+r} \int_{(\zeta, \lambda) \in \Gamma_{I} \times \Delta_{0 I}} f(\zeta) \wedge \bar{\partial}_{\zeta} K_{0 I}(z, \zeta, \lambda)
\end{aligned}
$$

et

$$
\begin{aligned}
\int_{\zeta \in S_{I \cup\{j\}}^{+}} f(\zeta) \wedge B_{I}(z, \zeta)= & \int_{(\zeta, \lambda) \in S_{I \cup\{j\}}^{+} \times \Delta_{0 I}} f(\zeta) \wedge K_{0 I}(z, \zeta, \lambda) \\
= & \int_{(\zeta, \lambda) \in \Gamma_{I} \cap\left\{\rho_{l} \geq 0\right\} \times \Delta_{0 I}} \bar{\partial} f(\zeta) \wedge K_{0 I}(z, \zeta, \lambda) \\
& +(-1)^{n+r} \int_{(\zeta, \lambda) \in \Gamma_{I} \cap\left\{\rho_{l} \geq 0\right\} \times \Delta_{0 I}} f(\zeta) \wedge \bar{\partial}_{\zeta} K_{0 I}(z, \zeta, \lambda) .
\end{aligned}
$$

Nous devons maintenant étudier avec plus de précision la singularité des noyaux $K_{0 I}$ et $\bar{\partial}_{\zeta} K_{0 I}$ en $z=\zeta$. Introduisons tout d'abord quelques notations.

DÉFINITION 3.5. Soit $I \in \mathcal{I}(l), 1 \leq l \leq k$, et $s$ un entier.

Une forme de type $O_{s}$ (ou de type $O_{s}(z, \zeta, \lambda)$ ) sur $\bar{D}_{I} \times \bar{D}_{I}^{*} \times \Delta_{0 I}$ est, par définition, une forme différentielle continue $f(z, \zeta, \lambda)$ définie pour tout $(z, \zeta, \lambda) \in$ $\bar{D}_{I} \times \bar{D}_{I}^{*} \times \Delta_{0 I}$ tel que $z \neq \zeta$ vérifiant les conditions suivantes.

1. Les dérivées d'ordre nul en $\zeta$, inférieur ou égal à 1 en $z$ et arbitraire en $\lambda$ des coefficients de $f$ sont continues pour tout $(z, \zeta, \lambda) \in \bar{D}_{I} \times \bar{D}_{I}^{*} \times \Delta_{0 I}$ tel que $z \neq \zeta$.

2. Soit $\nabla_{z}^{\kappa}, \kappa=0,1$, un opérateur différentiel à coefficients constants d'ordre nul en $\zeta$, inférieur ou égal à 1 en $z$ et arbitraire en $\lambda$. Il existe une constante $C>0$ telle que, pour chaque coefficient $\varphi(z, \zeta, \lambda)$ de $f(z, \zeta, \lambda)$,

$$
\left|\nabla_{z}^{\kappa} \varphi(z, \zeta, \lambda)\right| \leq C|\zeta-z|^{s-\kappa}
$$

pour tout $(z, \zeta, \lambda) \in \bar{D}_{I} \times \bar{D}_{I}^{*} \times \Delta_{0 I}$ tel que $z \neq \zeta$.

3. Il existe des voisinages $U_{0}$ et $U_{I}$ dans $\Delta_{0 I}$ de $\Delta_{0}$ et $\Delta_{I}$, respectivement, tels que $f(z, \zeta, \lambda)=0$ pour tout $(z, \zeta, \lambda) \in \bar{D}_{I} \times \bar{D}_{I}^{*} \times\left(U_{0} \cup U_{I}\right)$.

Les symboles $O_{s}$ et $O_{s}(z, \zeta, \lambda)$ sont utilisés de la manière suivante:

$f=O_{s}$ signifie $f$ est une forme de type $O_{s}$;

$O_{s} \wedge f=O_{k} \wedge g+O_{m}$ signifie pour toute forme $h$ de type $O_{s}$ il existe une forme $u$ de type $O_{k}$ et une forme $v$ de type $O_{m}$ telles que $h \wedge f=u \wedge g+v$. 
L’équation

$$
E f(z)=\int_{(\zeta, \lambda) \in S_{I} \times \Delta_{0 I}} O_{s}(z, \zeta, \lambda) \wedge f(z, \zeta, \lambda)
$$

signifie: il existe une forme $\hat{E}$ de type $O_{s}$ telle que

$$
E f(z)=\int_{(\zeta, \lambda) \in S_{I} \times \Delta_{0 I}} \hat{E}(z, \zeta, \lambda) \wedge f(z, \zeta, \lambda)
$$

pour toute $f$.

Rappelons que

$$
\psi_{0 I}(z, \zeta, \lambda)=\stackrel{\circ}{\chi}\left(\lambda_{0}\right) \frac{\bar{\zeta}-\bar{z}}{|\zeta-z|^{2}}=\left(1-\stackrel{\circ}{\chi}\left(\lambda_{0}\right)\right) \psi_{I}(z, \zeta, \stackrel{\circ}{\lambda})
$$

pour tout $I \in \mathcal{I}(l), 1 \leq l \leq k, z \in \bar{D}_{I}, \zeta \in \bar{D}_{I}^{*}$ tels que $z \neq \zeta$ et $\lambda \in \Delta_{0 I}$.

On pose

$$
\begin{aligned}
& W=W(z, \zeta, \stackrel{\circ}{)}=\langle w(z, \zeta, \stackrel{\circ}{\lambda}), d \zeta\rangle \quad \text { et } \\
& M=M(z, \zeta)=\frac{\langle\bar{\zeta}-\bar{z}, d \zeta\rangle}{|\zeta-z|^{2}}
\end{aligned}
$$

pour $z \in \bar{D}_{I}, \zeta \in \bar{D}_{I}^{*}$ tels que $z \neq \zeta$ et $\lambda \in \Delta_{0 I} \backslash \Delta_{0}$, où

$$
\begin{aligned}
\langle w(z, \zeta, \stackrel{\circ}{\lambda}), d \zeta\rangle & =\sum_{j=1}^{n} w_{j}(z, \zeta, \stackrel{\circ}{\lambda}) d \zeta_{j} \quad \text { et } \\
\langle\bar{\zeta}-\bar{z}, d \zeta\rangle & =\sum_{j=1}^{n}\left(\bar{\zeta}_{j}-\bar{z}_{j}\right) d \zeta_{j} .
\end{aligned}
$$

Soit $f$ une $(n, r)$-forme sur $\bar{D}$, on pose

$$
f(\zeta)=\tilde{f}(\zeta) d \zeta_{1} \wedge \cdots \wedge d \zeta_{n}
$$

Par Définition 3.3 des noyaux $K_{0 I}$ on a

$$
\begin{aligned}
f(\zeta) \wedge K_{0 I}(z, \zeta, \lambda)= & \frac{(-1)^{n(n-1) / 2}}{(2 i \pi)^{n}} \tilde{f}(\zeta) \wedge\left\langle\psi_{0 I}, d \zeta\right\rangle \\
& \wedge\left\langle\left(\bar{\partial}_{z, \zeta}+d_{\lambda}\right) \psi_{0 I}, d \zeta\right\rangle^{n-1} \wedge d z_{1} \wedge \cdots \wedge d z_{n}
\end{aligned}
$$

Alors, d'après la relation (3.11), on a

$$
\begin{aligned}
&\left\langle\psi_{0 I}, d \zeta\right\rangle=\stackrel{\circ}{\chi} M+(1-\stackrel{\circ}{\chi}) \frac{W}{\Phi} \\
&\left\langle\left(\bar{\partial}_{z, \zeta}+d_{\lambda}\right) \psi_{0 I}, d \zeta\right\rangle=\left(\frac{W}{\Phi}-M\right) \wedge d \stackrel{\circ}{\chi}+\stackrel{\circ}{\chi} \bar{\partial}_{z, \zeta} M+(1-\stackrel{\circ}{\chi}) \frac{\left(\bar{\partial}_{z, \zeta}+d_{\lambda}\right) W}{\Phi} \\
&+(1-\stackrel{\circ}{\chi}) \frac{W}{\Phi^{2}}\left(\bar{\partial}_{z, \zeta}+d_{\lambda}\right) \Phi .
\end{aligned}
$$


Comme on doit intégrer $f(\zeta) \wedge K_{0 I}(z, \zeta, \lambda)$ sur $\Delta_{0 I}$, qui est de dimension réelle $|I|$, seule la composante de degré $|I|$ en $\lambda$ du noyau $K_{0 I}$ donnera une contribution. Mais les formes différentielles $\left(\bar{\partial}_{z, \zeta}+d_{\lambda}\right) \Phi$ et $\left(\bar{\partial}_{z, \zeta}+d_{\lambda}\right) W$ sont les images réciproques de formes différentielles sur $\bar{D}_{I} \times \bar{D}_{I}^{*} \times \Delta_{I}$ par l'application $(z, \zeta, \lambda) \mapsto(z, \zeta, \lambda)$; par conséquent, puisque $\Delta_{I}$ est de dimension réelle $|I|-1$, pour tout $s=1,2, \ldots$, on a $\left[\left(\left(\bar{\partial}_{z, \zeta}+d_{\lambda}\right) W\right)^{s}\right]_{\operatorname{deg} \lambda=|I|}=0$ et $\left[\left(\left(\bar{\partial}_{z, \zeta}+d_{\lambda}\right) W\right)^{s} \wedge\right.$ $\left.\left(\bar{\partial}_{z, \zeta}+d_{\lambda}\right) \Phi\right]_{\operatorname{deg} \lambda=|I|}=0$. On a donc

$$
\begin{aligned}
& {\left[\left\langle\psi_{0 I}, d \zeta\right\rangle \wedge\left\langle\left(\bar{\partial}_{z, \zeta}+d_{\lambda}\right) \psi_{0 I}, d \zeta\right\rangle^{n-1}\right]_{\operatorname{deg} \lambda=|I|}} \\
& =\left(\stackrel{\circ}{\chi} M+(1-\stackrel{\circ}{\chi}) \frac{W}{\Phi}\right) \wedge(n-1)\left(\frac{W}{\Phi}-M\right) \wedge d \stackrel{\circ}{\chi} \\
& \wedge\left[\left(\stackrel{\circ}{\chi} \bar{\partial}_{z, \zeta} M+(1-\stackrel{\circ}{\chi}) \frac{\left(\bar{\partial}_{z, \zeta}+d_{\lambda}\right) W}{\Phi}\right.\right. \\
& \left.\left.+(1-\stackrel{\circ}{\chi}) \frac{W}{\Phi^{2}}\left(\bar{\partial}_{z, \zeta}+d_{\lambda}\right) \Phi\right)^{n-2}\right]_{\operatorname{deg} \lambda=|I|-1} .
\end{aligned}
$$

En développant l'expression précédente et en remarquant que $W \wedge W=0$ et $M \wedge M=0$, car $W$ et $M$ sont des 1-formes, on obtient

$$
\begin{aligned}
{\left[f(\zeta) \wedge K_{0 I}(z, \zeta, \lambda)\right]_{\operatorname{deg} \lambda=|I|}=} & a \tilde{f}(\zeta) \frac{M \wedge W}{\Phi} \wedge d \stackrel{\circ}{\chi} \\
& \wedge\left(\stackrel{\circ}{\chi} \bar{\partial}_{z, \zeta} M+(1-\stackrel{\circ}{\chi}) \frac{\bar{\partial}_{z, \zeta} W}{\Phi}\right)^{n-1-|I|} \\
& \wedge\left((1-\stackrel{\circ}{\chi}) \frac{d_{\lambda} W}{\Phi}\right)^{|I|-1},
\end{aligned}
$$

où $a$ désigne une constante.

Par définition des formes différentielles de type $O_{s}$, on a $d \stackrel{\circ}{\chi}=O_{0}, O_{0} \wedge M=$ $O_{-1}, O_{0} \wedge \bar{\partial}_{z, \zeta} M=O_{-2}$ et $O_{0} \wedge \bar{\partial}_{z, \zeta} W=O_{0}$, d'où

$$
\left[f(\zeta) \wedge K_{0 I}(z, \zeta, \lambda)\right]_{\operatorname{deg} \lambda=|I|}=\frac{O_{-1} \wedge W}{\Phi^{|I|}} \wedge\left(O_{-2}+\frac{O_{0}}{\Phi}\right)^{n-1-|I|} \wedge\left(d_{\lambda} W\right)^{|I|-1} .
$$

Par ailleurs on a

$$
\begin{aligned}
O_{0} \wedge W & =\sum_{j \in I} O_{0} \wedge \partial \rho_{j}(\zeta)+O_{1}, \\
O_{0} \wedge d_{\lambda} W & =\sum_{j \in I} O_{0} \wedge \partial \rho_{j}(\zeta)+O_{1}, \\
O_{0} \wedge W \wedge\left(d_{\lambda} W\right)^{|I|-1} & =\sum_{\substack{0 \leq m \leq|I| \\
i_{1}, \ldots, i_{m} \in I}} O_{|I|-m} \wedge \partial \rho_{i_{1}}(\zeta) \wedge \cdots \wedge \partial \rho_{i_{m}}(\zeta),
\end{aligned}
$$

et par conséquent 
$\left[f(\zeta) \wedge K_{0 I}(z, \zeta, \lambda)\right]_{\operatorname{deg} \lambda=|I|}$

$$
=\sum_{\substack{0 \leq s \leq n-1-|I| \\ 0 \leq m \leq|I|}} \frac{O_{|I|-2 n+2(|I|+s-m)+m+1}}{\Phi^{(|I|+s-m)+m}} \wedge \partial \rho_{i_{1}}(\zeta) \wedge \cdots \wedge \partial \rho_{i_{m}}(\zeta) .
$$

En différentiant $K_{0 I}$ par rapport à $\bar{\zeta}$, on obtient

$$
\begin{aligned}
& {\left[f(\zeta) \wedge \bar{\partial}_{\zeta} K_{0 I}(z, \zeta, \lambda)\right]_{\operatorname{deg} \lambda=|I|}} \\
& =a \tilde{f}(\zeta)\left(\frac{\bar{\partial}_{\zeta} M \wedge W}{\Phi^{|I|}}-\frac{M \wedge \bar{\partial}_{\zeta} W}{\Phi^{|I|}}+\frac{M \wedge W}{\Phi^{|I|+1}} \wedge \bar{\partial}_{\zeta} \Phi\right) \wedge d \stackrel{\circ}{\chi} \\
& \wedge\left(\stackrel{\circ}{\chi} \bar{\partial}_{z, \zeta} M+(1-\stackrel{\circ}{\chi}) \frac{\bar{\partial}_{z, \zeta} W}{\Phi}\right)^{n-1-|I|} \wedge\left((1-\stackrel{\circ}{\chi}) d_{\lambda} W\right)^{|I|-1} \\
& +(n-1-|I|) a \tilde{f}(\zeta) \frac{M \wedge W}{\Phi^{|I|}} \wedge d \stackrel{\circ}{\chi} \\
& \wedge\left(\stackrel{\circ}{\chi} \bar{\partial}_{\zeta} \bar{\partial}_{z} M+(1-\stackrel{\circ}{\chi}) \frac{\bar{\partial}_{\zeta} \bar{\partial}_{z} W}{\Phi}+(1-\stackrel{\circ}{\chi}) \frac{\bar{\partial}_{z, \zeta} W \wedge \bar{\partial}_{\zeta} \Phi}{\Phi^{2}}\right) \\
& \wedge\left(\stackrel{\circ}{\chi}_{\bar{\partial}} \bar{z}_{z, \zeta} M+(1-\stackrel{\circ}{\chi}) \frac{\bar{\partial}_{z, \zeta} W}{\Phi}\right)^{n-2-|I|} \wedge\left((1-\stackrel{\circ}{\chi}) d_{\lambda} W\right)^{|I|-1} \\
& +(|I|-1) a \tilde{f}(\zeta) \frac{M \wedge W}{\Phi^{|I|}} \wedge d \stackrel{\circ}{\chi} \\
& \wedge\left(\stackrel{\circ}{\chi} \bar{\partial}_{z, \zeta} M+(1-\stackrel{\circ}{\chi}) \frac{\bar{\partial}_{z, \zeta} W}{\Phi}\right)^{n-1-|I|} \wedge(1-\stackrel{\circ}{\chi})^{|I|-1} \bar{\partial}_{\zeta} d_{\lambda} W \wedge\left(d_{\lambda} W\right)^{|I|-2}
\end{aligned}
$$

ce qui donne

$$
\left[f(\zeta) \wedge \bar{\partial}_{\zeta} K_{0 I}(z, \zeta, \lambda)\right]_{\operatorname{deg} \lambda=|I|}=A+B+C+D+E+F+G
$$

avec

$$
\begin{aligned}
& A=\frac{O_{-2}}{\Phi^{|I|}} \wedge\left(O_{-2}+\frac{O_{0}}{\Phi}\right)^{n-1-|I|} \wedge W \wedge\left(d_{\lambda} W\right)^{|I|-1} \\
& B=\frac{O_{-1}}{\Phi^{|I|}} \wedge\left(O_{-2}+\frac{O_{0}}{\Phi}\right)^{n-1-|I|} \wedge\left(d_{\lambda} W\right)^{|I|-1}, \\
& C=\frac{O_{-1}}{\Phi^{|I|+1}} \wedge\left(O_{-2}+\frac{O_{0}}{\Phi}\right)^{n-1-|I|} \wedge W \wedge\left(d_{\lambda} W\right)^{|I|-1} \wedge \bar{\partial}_{\zeta} \Phi \\
& D=\frac{O_{-4}}{\Phi^{|I|}} \wedge\left(O_{-2}+\frac{O_{0}}{\Phi}\right)^{n-2-|I|} \wedge W \wedge\left(d_{\lambda} W\right)^{|I|-1}, \\
& E=\frac{O_{-1}}{\Phi^{|I|+1}} \wedge\left(O_{-2}+\frac{O_{0}}{\Phi}\right)^{n-2-|I|} \wedge W \wedge\left(d_{\lambda} W\right)^{|I|-1},
\end{aligned}
$$




$$
\begin{aligned}
& F=\frac{O_{-1}}{\Phi^{|I|+2}} \wedge\left(O_{-2}+\frac{O_{0}}{\Phi}\right)^{n-2-|I|} \wedge W \wedge\left(d_{\lambda} W\right)^{|I|-1} \wedge \bar{\partial}_{\zeta} \Phi \\
& G=\frac{O_{-1}}{\Phi^{|I|}} \wedge\left(O_{-2}+\frac{O_{0}}{\Phi}\right)^{n-1-|I|} \wedge W \wedge \bar{\partial}_{\zeta} d_{\lambda} W \wedge\left(d_{\lambda} W\right)^{|I|-2}
\end{aligned}
$$

\section{On en déduit}

$$
\begin{aligned}
& A=\sum_{\substack{0 \leq s \leq n-1-|I| \\
0 \leq m \leq|I|}} \frac{O_{|I|-2 n+2(|I|+s-m)+m}}{\Phi^{(|I|+s-m)+m}} \wedge \partial \rho_{i_{1}}(\zeta) \wedge \cdots \wedge \partial \rho_{i_{m}}(\zeta), \\
& B=\sum_{\substack{0 \leq s \leq n-1-|I| \\
0 \leq m \leq|I|-1}} \frac{O_{|I|-2 n+2(|I|+s-m)+m}}{\Phi^{(|I|+s-m)+m}} \wedge \partial \rho_{i_{1}}(\zeta) \wedge \cdots \wedge \partial \rho_{i_{m}}(\zeta), \\
& C=\sum_{\substack{0 \leq s \leq n-1-|I| \\
0 \leq m \leq|I|}} \frac{O_{|I|-2 n+2(|I|+1+s-m)+m}}{\Phi^{(|I|+1+s-m)+m}} \wedge \partial \rho_{i_{1}}(\zeta) \wedge \cdots \wedge \partial \rho_{i_{m}}(\zeta), \\
& +\sum_{\substack{0 \leq s \leq n-1-|I| \\
0 \leq m \leq|I|+1}} \frac{O_{|I|-2 n+2(|I|+1+s-m)+m}}{\Phi^{(|I|+1+s-m)+m}} \wedge \bar{\partial} \rho_{i_{1}} \wedge \partial \rho_{i_{2}}(\zeta) \wedge \cdots \wedge \partial \rho_{i_{m}}(\zeta), \\
& D=\sum_{\substack{0 \leq s \leq n-2-|I| \\
0 \leq m \leq|I|}} \frac{O_{|I|-2 n+2(|I|+s-m)+m}}{\Phi^{(|I|+s-m)+m}} \wedge \partial \rho_{i_{1}}(\zeta) \wedge \cdots \wedge \partial \rho_{i_{m}}(\zeta), \\
& E=\sum_{\substack{0 \leq s \leq n-2-|I| \\
0 \leq m \leq|I|}} \frac{O_{|I|-2 n+2(|I|+1+s-m)+m+1}}{\Phi^{(|I|+1+s-m)+m}} \wedge \partial \rho_{i_{1}}(\zeta) \wedge \cdots \wedge \partial \rho_{i_{m}}(\zeta), \\
& F=\sum_{\substack{0 \leq s \leq n-2-|I| \\
0 \leq m \leq|I|}} \frac{O_{|I|-2 n+2(|I|+2+s-m)+m}}{\Phi^{(|I|+2+s-m)+m}} \wedge \partial \rho_{i_{1}}(\zeta) \wedge \cdots \wedge \partial \rho_{i_{m}}(\zeta), \\
& +\sum_{\substack{0 \leq s \leq n-2-|I| \\
0 \leq m \leq|I|+1}} \frac{O_{|I|-2 n+2(|I|+2+s-m)+m}}{\Phi^{(|I|+2+s-m)+m}} \wedge \bar{\partial} \rho_{i_{1}} \wedge \partial \rho_{i_{2}}(\zeta) \wedge \cdots \wedge \partial \rho_{i_{m}}(\zeta), \\
& G=\sum_{\substack{0 \leq s \leq n-1-|I| \\
0 \leq m \leq|I|-1}} \frac{O_{|I|-2 n+2(|I|+s-m)+m}}{\Phi^{(|I|+s-m)+m}} \wedge \partial \rho_{i_{1}}(\zeta) \wedge \cdots \wedge \partial \rho_{i_{m}}(\zeta),
\end{aligned}
$$

DÉfinition 3.6. Soient $m \in \mathbb{N}$, un entier, et $I \in \mathcal{I}(l), 1 \leq l \leq k$, un multi-indice. Un opérateur de type $m$ est, par définition, une application

$$
E: \mathcal{C}_{n, *}(D) \rightarrow \mathcal{C}_{n, *}^{\infty}\left(D_{I}\right)
$$


telle qu'il existe

- un entier $\kappa \geq 0$,

- une forme différentielle $\hat{E}(z, \zeta, \lambda)$ de type $O_{|I|-2 n+2 \kappa+m}$ sur $D_{I} \times \Gamma_{I} \times \Delta_{0 I}$ telle que pour toute forme différentielle $f \in \mathcal{C}_{n, *}(D)$

$$
E f(z)=\int_{(\zeta, \lambda) \in \Gamma_{I} \times \Delta_{0 I}} \tilde{f}(\zeta) \wedge \frac{\hat{E}(z, \zeta, \lambda) \wedge \Theta(\zeta)}{\Phi^{\kappa+m}(z, \zeta, \stackrel{\circ}{\lambda})}
$$

où $\tilde{f} \in \mathcal{C}_{0, *}(D)$ est la forme différentielle définie par

$$
\begin{gathered}
f(\zeta)=\tilde{f}(\zeta) d \zeta_{1} \wedge \cdots \wedge d \zeta_{n}, \\
\Theta=1, \text { si } m=0, \text { et } \Theta=\partial \rho_{i_{1}}(\zeta) \wedge \cdots \wedge \partial \rho_{i_{m}}(\zeta) \text { ou } \Theta=\bar{\partial} \rho_{i_{1}} \wedge \partial \rho_{i_{2}}(\zeta) \wedge \cdots \wedge \\
\partial \rho_{i_{m}}(\zeta), \text { si } m \geq 1, \text { avec } i_{1}, \ldots, i_{m} \in I
\end{gathered}
$$

Les calculs précédents montrent alors immédiatement la proposition suivante:

Proposition 3.7. Les opérateurs intégraux

$$
\begin{aligned}
& f \mapsto \int_{\zeta \in S_{I}} f(\zeta) \wedge B_{I}(z, \zeta) \quad e t \\
& f \mapsto \int_{\zeta \in S_{I \cup\{j\}}^{+}} f(\zeta) \wedge B_{I}(z, \zeta)
\end{aligned}
$$

sont des sommes finies d'opérateurs de type $m$.

Il résulte alors de l'étude faite dans [16] que pour tout $I \in \mathcal{I}(l), 1 \leq l \leq k$, tout $j \in\{ \pm 1, \ldots, \pm k\}$ tel que $I \cup\{j\} \in \mathcal{I}$ et toute forme différentielle $\bar{f}$ de classe $\mathcal{C}^{1}$ sur $U$ à support dans $D$, les formes différentielles $\int_{\zeta \in S_{I}} f(\zeta) \wedge B_{I}(z, \zeta)$ et $\int_{\zeta \in S_{I \cup\{j\}}^{+}} f(\zeta) \wedge B_{I}(z, \zeta)$ sont de classe $\mathcal{C}^{1 / 2-\varepsilon}$ pour tout $\varepsilon>0$ et donc continues sur $\bar{D}_{I}$ et de classe $\mathcal{C}^{\infty}$ sur $D_{I}$.

Soit $\chi$ une fonction de classe $\mathcal{C}^{\infty}$ sur $\mathbb{R}$ à valeurs dans $[0,1]$ telle que $\chi(x)=1$ si $|x| \leq 1 / 2$ et $\chi(x)=0$ si $|x| \geq 1$. On pose $\chi_{\varepsilon}(z, \zeta)=\chi(|\zeta-z| / \varepsilon)$.

LeMme 3.8. Pour tout $z \in S_{I}$ et toute forme différentielle $f$ de classe $\mathcal{C}^{1}$ sur $U$ à support dans $D$, on a

$$
\int_{\zeta \in S_{I}} f(\zeta) \wedge B_{I}(z, \zeta)=\lim _{\varepsilon \rightarrow 0} \int_{\zeta \in S_{I}}\left(1-\chi_{\varepsilon}(z, \zeta)\right) f(\zeta) \wedge B_{I}(z, \zeta)
$$

Démonstration. Soit $z \in S_{I}$, comme $1-\chi_{\varepsilon}(z, \zeta)=0$ au voisinage de $z$, il résulte de la formule de Stokes que 


$$
\begin{aligned}
\int_{\zeta \in S_{I}}(1 & \left.-\chi_{\varepsilon}(z, \zeta)\right) f(\zeta) \wedge B_{I}(z, \zeta) \\
= & \int_{(\zeta, \lambda) \in \Gamma_{I} \times \Delta_{0 I}} \bar{\partial}_{\zeta}\left(\left(1-\chi_{\varepsilon}(z, \zeta)\right) f(\zeta)\right) \wedge K_{0 I}(z, \zeta, \lambda) \\
& +(-1)^{n+r} \int_{(\zeta, \lambda) \in \Gamma_{I} \times \Delta_{0 I}}\left(1-\chi_{\varepsilon}(z, \zeta)\right) f(\zeta) \wedge \bar{\partial}_{\zeta} K_{0 I}(z, \zeta, \lambda) \\
= & \int_{(\zeta, \lambda) \in \Gamma_{I} \times \Delta_{0 I}} \bar{\partial}_{\zeta} f(\zeta) \wedge K_{0 I}(z, \zeta, \lambda) \\
& +(-1)^{n+r} \int_{(\zeta, \lambda) \in \Gamma_{I} \times \Delta_{0 I}} f(\zeta) \wedge \bar{\partial}_{\zeta} K_{0 I}(z, \zeta, \lambda) \\
& -\int_{(\zeta, \lambda) \in \Gamma_{I} \times \Delta_{0 I}} \bar{\partial}_{\zeta}\left(\chi_{\varepsilon}(z, \zeta) f(\zeta)\right) \wedge K_{0 I}(z, \zeta, \lambda) \\
& -(-1)^{n+r} \int_{(\zeta, \lambda) \in \Gamma_{I} \times \Delta_{0 I}} \chi_{\varepsilon}(z, \zeta) f(\zeta) \wedge \bar{\partial}_{\zeta} K_{0 I}(z, \zeta, \lambda)
\end{aligned}
$$

Mais d'après l'étude précédente, $\int_{\zeta \in S_{I}} f(\zeta) \wedge B_{I}(z, \zeta)$ est continue sur $S_{I}$ et

$$
\begin{aligned}
\int_{\zeta \in S_{I}} f(\zeta) \wedge B_{I}(z, \zeta)= & \int_{(\zeta, \lambda) \in \Gamma_{I} \times \Delta_{0 I}} \bar{\partial}_{\zeta} f(\zeta) \wedge K_{0 I}(z, \zeta, \lambda) \\
& +(-1)^{n+r} \int_{(\zeta, \lambda) \in \Gamma_{I} \times \Delta_{0 I}} f(\zeta) \wedge \bar{\partial}_{\zeta} K_{0 I}(z, \zeta, \lambda)
\end{aligned}
$$

Nous devons donc prouver que, pour tout $z \in S_{I}$ et toute forme différentielle $f$ de classe $\mathcal{C}^{1}$ sur $U$ à support dans $D$,

$$
\begin{aligned}
\lim _{\varepsilon \rightarrow 0} \int_{(\zeta, \lambda) \in \Gamma_{I} \times \Delta_{0 I}} & \bar{\partial}_{\zeta}\left(\chi_{\varepsilon}(z, \zeta) f(\zeta)\right) \wedge K_{0 I}(z, \zeta, \lambda) \\
& +(-1)^{n+r} \int_{(\zeta, \lambda) \in \Gamma_{I} \times \Delta_{0 I}} \chi_{\varepsilon}(z, \zeta) f(\zeta) \wedge \bar{\partial}_{\zeta} K_{0 I}(z, \zeta, \lambda)=0
\end{aligned}
$$

Grâce aux estimations des noyaux $K_{0 I}$ et $\bar{\partial}_{\zeta} K_{0 I}$, et par définition de $\chi_{\varepsilon}$, il suffit d'évaluer des intégrales du type suivant:

$$
\begin{aligned}
& A_{I, \varepsilon}(f)=\int_{(\zeta, \lambda) \in \Gamma_{I} \times \Delta_{0 I}} \bar{\partial}_{\zeta}\left(\chi_{\varepsilon}(z, \zeta) f(\zeta)\right) \wedge \frac{O_{|I|-2 n+2 \kappa+m+1}(z, \zeta, \lambda) \wedge \Theta(\zeta)}{\Phi^{\kappa+m}(z, \zeta, \grave{\lambda})}, \\
& C_{I, \varepsilon}(f)=\int_{(\zeta, \lambda) \in \Gamma_{I} \times \Delta_{0 I}} \chi_{\varepsilon}(z, \zeta) f(\zeta) \wedge \frac{O_{|I|-2 n+2 \kappa+m}(z, \zeta, \lambda) \wedge \Theta(\zeta)}{\Phi^{\kappa+m}(z, \zeta, \stackrel{\circ}{\lambda})} .
\end{aligned}
$$

On déduit des résultats des paragraphes 6 et 7 de [16] et de l'inégalité (3.8) qu'après intégration partielle en $\lambda$, les intégrales précédentes sont contrôlées par des sommes finies d'intégrales de la forme: 


$$
\begin{aligned}
N_{I, \varepsilon} & =\frac{1}{\varepsilon} \int_{\zeta \in \Gamma_{I} \cap B(z, \varepsilon)} \frac{\left|\sigma \wedge d \rho_{I}\right|}{\left(\left|\rho_{I}\right|+|\zeta-z|^{2}\right)|\zeta-z|^{2 n-|I|-2}}, \\
M_{I, \varepsilon} & =\int_{\zeta \in \Gamma_{I} \cap B(z, \varepsilon)} \frac{\left|\sigma \wedge d \rho_{I}\right|}{\left(\left|\rho_{I}\right|+|\zeta-z|^{2}\right)|\zeta-z|^{2 n-|I|-1}},
\end{aligned}
$$

et, pour $1 \leq s \leq|I|$,

$$
\begin{aligned}
N_{I, \varepsilon} & =\frac{1}{\varepsilon} \int_{\zeta \in \Gamma_{I} \cap B(z, \varepsilon)} \frac{\left|\sigma \wedge d \rho_{I} \bigwedge_{\nu=1}^{s} d t_{\nu}\right|}{\left(\left|\rho_{I}\right|+|\zeta-z|^{2}\right) \prod_{\nu=1}^{s}\left(\left|t_{\nu}\right|+|\zeta-z|^{2}\right)|\zeta-z|^{2 n-|I|-s-2}}, \\
M_{I, \varepsilon} & =\int_{\zeta \in \Gamma_{I} \cap B(z, \varepsilon)} \frac{\left|\sigma \wedge d \rho_{I} \bigwedge_{\nu=1}^{s} d t_{\nu}\right|}{\left(\left|\rho_{I}\right|+|\zeta-z|^{2}\right) \prod_{\nu=1}^{s}\left(\left|t_{\nu}\right|+|\zeta-z|^{2}\right)|\zeta-z|^{2 n-|I|-s-1}},
\end{aligned}
$$

où $\rho_{I}$ est la fonction définie $\operatorname{par} \rho_{I}(\zeta)=\rho_{i_{1}}(\zeta)=\cdots=\rho_{i_{|I|}}(\zeta)$ pour $\zeta \in \Gamma_{I}$, $\sigma$ un monôme en $d \zeta_{1}, \ldots, d \zeta_{n}, d \bar{\zeta}_{1}, \ldots, d \bar{\zeta}_{n}, \lambda^{1}, \ldots, \lambda^{|I|}$ des points de $\Delta_{I}$ qui forment un système de vecteurs indépendants de $\mathbb{R}^{|I|}, t_{v}=\operatorname{Im} \Phi\left(z, \zeta, \lambda^{v}\right)$ et $d t_{v}=$ $d_{\zeta} \operatorname{Im} \Phi\left(z, \zeta, \lambda^{\nu}\right)$.

La fonction $\rho_{I}$ peut être utilisée comme coordonnée locale sur $\Gamma_{I}$ ainsi que les fonctions $t_{v}, 1 \leq v \leq s$, car $M$ est générique, par conséquent

$$
\begin{aligned}
N_{I, \varepsilon} & \lesssim \frac{1}{\varepsilon} \int_{\substack{X \in \mathbb{R}^{2 n-|I|+1} \\
\prod_{1<0,|X|<\varepsilon}^{s}\left(\left|X_{i}\right|+|X|^{2}\right)|X|^{2 n-|I|-s-2}}} \\
& \lesssim \varepsilon(1+|\ln \varepsilon|)^{s+1}
\end{aligned}
$$

et

$$
\begin{aligned}
M_{I, \varepsilon} & \lesssim \int_{\substack{X \in \mathbb{R}^{2 n-|I|+1} \\
\prod_{1<0,|X|<\varepsilon}}} \frac{d X}{\prod_{i=1}^{s}\left(\left|X_{i}\right|+|X|^{2}\right)|X|^{2 n-|I|-s-1}} \\
& \lesssim \varepsilon(1+|\ln \varepsilon|)^{s+1}
\end{aligned}
$$

et donc $\lim _{\varepsilon \rightarrow 0} N_{I, \varepsilon}=\lim _{\varepsilon \rightarrow 0} M_{I, \varepsilon}=0$.

Considérons à présent la dernière condition d'intégrabilité:

- $\operatorname{pour} I=J$ ou $I=\delta(K)$ et $J=K(\widehat{|K|})$ avec $K \in \mathcal{I}(l+1), \int_{\zeta \in \tilde{S}_{J}} f_{\varepsilon}(\zeta) \wedge B_{I}(z, \zeta)$ tend vers 0 , quand $\varepsilon$ tend vers 0 , si $f_{\varepsilon}$ est une forme différentielle continue sur $U$ à support dans $D \cap\left\{\left|\rho_{1}\right|<\varepsilon\right\} \cap \cdots \cap\left\{\left|\rho_{k}\right|<\varepsilon\right\}$ telle que $\left\|\bar{\partial} f_{\varepsilon}\right\|=O(1 / \varepsilon)$ et $\int_{\zeta \in \tilde{S}_{J}} f_{\varepsilon}(\zeta) \wedge B_{I}(z, \zeta)=o(\varepsilon)$, si de plus $\bar{\partial} f_{\varepsilon}=0$.

On note $\tilde{\Gamma}_{J}$ la partie de $\Gamma_{J}$, qui vérifie $\partial \tilde{\Gamma}_{J} \cap S_{J}=\tilde{S}_{J}$. Par la formule de Stokes et les résultats de continuité précédents, on obtient pour $z \in \bar{D}_{I}$

$$
\begin{aligned}
\int_{\zeta \in \tilde{S}_{J}} f_{\varepsilon}(\zeta) \wedge B_{I}(z, \zeta)= & \int_{(\zeta, \lambda) \in \tilde{\Gamma}_{J} \times \Delta_{0 J}} f_{\varepsilon}(\zeta) \wedge \bar{\partial}_{\zeta} K_{0 I}(z, \zeta, \lambda) \\
& +(-1)^{n+r} \int_{(\zeta, \lambda) \in \tilde{\Gamma}_{J} \times \Delta_{0 J}} \bar{\partial} f_{\varepsilon}(\zeta) \wedge K_{0 I}(z, \zeta, \lambda) .
\end{aligned}
$$

Grâce aux estimations que nous venons d'effectuer, pour évaluer chaque terme du second membre de la relation précédente, nous sommes amenés à étudier des intégrales du type suivant: 


$$
A_{I, J} \varphi_{\varepsilon}(z)=\int_{(\zeta, \lambda) \in \tilde{\Gamma}_{J, \varepsilon} \times \Delta_{0 J}} \varphi_{\varepsilon}(\zeta) \frac{O_{|I|-2 n+2 \kappa+m}(z, \zeta, \lambda) \wedge \Theta(\zeta)}{\Phi^{\kappa+m}(z, \zeta, \stackrel{\circ}{\lambda})}
$$

où $\tilde{\Gamma}_{J, \varepsilon}=\tilde{\Gamma}_{J} \cap\left\{\left|\rho_{1}\right|<\varepsilon\right\} \cap \cdots \cap\left\{\left|\rho_{k}\right|<\varepsilon\right\}$ et $\varphi_{\varepsilon}$ désigne soit $\tilde{f}_{\varepsilon}$, soit $\bar{\partial} \tilde{f}_{\varepsilon}$.

Les résultats des paragraphes 6 et 7 de [16] nous disent qu'après intégration partielle en $\lambda$ les intégrales $A_{I, J} \varphi_{\varepsilon}(z)$ sont contrôlées par des sommes finies d'intégrales du type suivant:

$$
M_{J} \varphi_{\varepsilon}(z)=\int_{\zeta \in \tilde{\Gamma}_{J, \varepsilon}} \frac{\left\|\varphi_{\varepsilon}\right\|(\zeta)\left|\sigma \wedge d \rho_{J}\right|}{\left(\left|\rho_{J}\right|+d+|\zeta-z|^{2}\right)|\zeta-z|^{2 n-|J|-1}}
$$

et, pour $1 \leq s \leq|J|$,

$M_{J} \varphi_{\varepsilon}(z)$

$$
=\int_{\zeta \in \tilde{\Gamma}_{J, \varepsilon}} \frac{\left\|\varphi_{\varepsilon}\right\|(\zeta)\left|\sigma \wedge d \rho_{J} \bigwedge_{\nu=1}^{s} d t_{\nu}\right|}{\left(\left|\rho_{J}\right|+d+|\zeta-z|^{2}\right) \prod_{\nu=1}^{s}\left(\left|t_{\nu}\right|+d+|\zeta-z|^{2}\right)|\zeta-z|^{2 n-|J|-s-1}},
$$

où $\rho_{J}$ est la fonction définie $\operatorname{par} \rho_{J}(\zeta)=\rho_{j_{1}}(\zeta)=\cdots=\rho_{j_{|J|}}(\zeta) \operatorname{pour} \zeta \in \Gamma_{J}, d$ la distance de $z$ à $S_{J}, \sigma$ un monôme en $d \zeta_{1}, \ldots, d \zeta_{n}, d \bar{\zeta}_{1}, \ldots, d \bar{\zeta}_{n}, \lambda^{1}, \ldots, \lambda^{s}$ des points de $\Delta_{J}, t_{v}=\operatorname{Im} \Phi\left(z, \zeta, \lambda^{\nu}\right)$ et $d t_{v}=d_{\zeta} \operatorname{Im} \Phi\left(z, \zeta, \lambda^{\nu}\right)$.

La fonction $\rho_{J}$ peut être utilisée comme coordonnée locale sur $\Gamma_{J}$ et $\tilde{\Gamma}_{J, \varepsilon}$ peut être paramétré localement par $\left\{\left(X^{\prime}, \rho_{J}, X^{\prime \prime}\right) \in \mathbb{R}^{2 n-k} \times \mathbb{R} \times \mathbb{R}^{k-|J|}|| X^{\prime} \mid \leq C\right.$, $\left.0 \leq \rho_{J} \leq \varepsilon, X^{\prime \prime}=\left(Y^{\prime}, Y\right), Y<\left|X^{\prime \prime}\right|^{2}\right\}$. On en déduit en passant en coordonnées cylindriques que

$$
M_{J} \varphi_{\varepsilon}(z) \lesssim\left\|\varphi_{\varepsilon}\right\| \varepsilon^{2}(1+|\ln \varepsilon|)
$$

et, pour $1 \leq s \leq|J|$,

$$
M_{J}^{\prime} \varphi_{\varepsilon}(z) \lesssim\left\|\varphi_{\varepsilon}\right\| \varepsilon^{2}(1+|\ln \varepsilon|)^{1+s} .
$$

Ce qui prouve la dernière condition d'intégrabilité.

\section{Une Deuxième Solution Fondamentale}

On considère toujours une sous-variété CR générique, $q$-concave $M$ de classe $\mathcal{C}^{3}$, de codimension réelle $k$ dans $\mathbb{C}^{n}$ et un point $z_{0}$ de $M$. Il résulte du Théorème 2.5 que le noyau $B_{M}=\sum_{I \in \mathcal{I}^{\prime}(k)} \operatorname{sgn}(I) B_{I}$, obtenu à partir des noyaux $B_{I}$ construits dans la Section 3, est une solution fondamentale de l'opérateur de Cauchy-Riemann tangentiel sur un voisinage $U_{z_{0}}$ de $z_{0}$ pour certains bidegrés, plus précisément c'est une forme différentielle définie sur $U_{z_{0}} \times U_{z_{0}} \backslash \Delta\left(U_{z_{0}}\right)$, où $\Delta\left(U_{z_{0}}\right)=\{(z, \zeta) \in$ $\left.U_{z_{0}} \times U_{z_{0}} \mid z=\zeta\right\}$ désigne la diagonale de $U_{z_{0}} \times U_{z_{0}}$, qui vérifie au sens des courants

$$
\bar{\partial}_{z}\left[B_{M}\right]_{p, r-1}+\bar{\partial}_{\zeta}\left[B_{M}\right]_{p, r}=(-1)^{k(k+1) / 2}\left[\Delta\left(U_{z_{0}}\right)\right],
$$

où $\left[B_{M}\right]_{p, r}$ désigne la composante de bidegré $(p, r)$ en $z$ de $B_{M}, 0 \leq p \leq n$ et $n-k-q+1 \leq r \leq n-k, \bar{\partial}_{z, \zeta}=\bar{\partial}_{z}+\bar{\partial}_{\zeta}$ l'opérateur de Cauchy-Riemann tangentiel sur $M \times M$ et $\left[\Delta\left(U_{z_{0}}\right)\right]$ le courant d'intégration sur la diagonale de $U_{z_{0}} \times U_{z_{0}}$. L'objet de cette section est de construire une nouvelle solution fondamentale de 
l'opérateur de Cauchy-Riemann tangentiel au voisinage de $z_{0}$ dont la singularité sur la diagonale est d'ordre inférieur.

Pour tout $I \in \mathcal{I}^{\prime}(k)$, on note $I *$ le multi-indice $\left(i_{1}, \ldots, i_{k}, *\right)$, où $I=\left(i_{1}, \ldots, i_{k}\right)$, et $\mathcal{I}^{\prime}(k, *)$ l'ensemble des multi-indices $I *$, lorsque $I$ décrit $\mathcal{I}^{\prime}(k)$. On pose $\rho_{*}=$ $\rho_{1}+\cdots+\rho_{k}$ et $\rho_{\lambda}=\lambda_{i_{1}} \rho_{i_{1}}+\cdots+\lambda_{i_{k}} \rho_{i_{k}}+\lambda_{*} \rho_{*}$ pour $\lambda=\left(\lambda_{i_{1}}, \ldots, \lambda_{i_{k}}, \lambda_{*}\right) \in$ $\Delta_{I *}$.

La variété $M$ étant $q$-concave, il résulte de la définition des fonctions définissantes $\rho_{1}, \ldots, \rho_{k}$ qu'il existe un sous espace vectoriel $E_{*}$ de dimension $q+k$ sur lequel la forme de Levi de $\rho_{*}$ sur $U$ est définie positive.

Comme dans la Section 3 on construit des fonctions $w^{*}$ et $\Phi_{*}$ pour la fonction $\rho_{*}$. Soit

$$
F_{*}(z, \zeta)=2 \sum_{j=1}^{n} \frac{\partial \rho_{*}}{\partial \zeta_{j}}(\zeta)\left(\zeta_{j}-z_{j}\right)-\sum_{j, k=1}^{n} \frac{\partial^{2} \rho_{*}}{\partial \zeta_{j} \partial \zeta_{k}}(\zeta)\left(\zeta_{j}-z_{j}\right)\left(\zeta_{k}-z_{k}\right)
$$

Si $Q^{*}$ désigne la projection orthogonale sur le supplémentaire orthogonal du sous espace $E_{*}$, la formule de Taylor implique qu'il existe des constantes strictement positives $\alpha^{*}$ et $B$ telles que

$$
\operatorname{Re} F_{*}(z, \zeta) \geq \rho_{*}(\zeta)-\rho_{*}(z)+\alpha|\zeta-z|^{2}-B\left|Q^{*}(\zeta-z)\right|^{2}
$$

pour $\zeta, z$ au voisinage de $\bar{D}$.

On pose alors

$$
\begin{aligned}
& w_{j}^{*}(z, \zeta)=2 \frac{\partial \rho_{*}}{\partial \zeta_{j}}(\zeta)-\sum_{k=1}^{n} a_{j k}^{*}(\zeta)\left(\zeta_{k}-z_{k}\right)+B \sum_{k=1}^{n} \overline{Q_{j k}^{*}\left(\zeta_{k}-z_{k}\right)}, \\
& w^{*}(z, \zeta)=\left(w_{1}^{*}(z, \zeta), \ldots, w_{n}^{*}(z, \zeta)\right), \\
& \Phi_{*}(z, \zeta)=\left\langle w^{*}(z, \zeta), \zeta-z\right\rangle
\end{aligned}
$$

où les fonctions $a_{j k}^{*}, j, k=1, \ldots, n$, sont de classe $\mathcal{C}^{\infty}$ sur $U$ et vérifient pour tout $\zeta \in U$

$$
\left|a_{j k}^{*}(\zeta)-\frac{\partial^{2} \rho_{*}}{\partial \zeta_{j} \partial \zeta_{k}}(\zeta)\right|<\frac{\alpha^{*}}{2 n^{2}}
$$

Soit

$$
\tilde{F}_{*}(\zeta, z)=2 \sum_{j=1}^{n} \frac{\partial \rho_{*}}{\partial \zeta_{j}}(\zeta)\left(\zeta_{j}-z_{j}\right)-\sum_{j, k=1}^{n} a_{j k}^{*}(\zeta)\left(\zeta_{j}-z_{j}\right)\left(\zeta_{k}-z_{k}\right),
$$

alors

$$
\Phi_{*}(z, \zeta)=\tilde{F}_{*}(\zeta, z)+B\left|Q^{*}(\zeta-z)\right|^{2}
$$

et par conséquent

$$
\operatorname{Re} \Phi_{*}(z, \zeta) \geq \rho_{*}(\zeta)-\rho_{*}(z)+\frac{\alpha^{*}}{2}|\zeta-z|^{2} .
$$

Si $\lambda=\left(\lambda_{i_{1}}, \ldots, \lambda_{i_{k}}, \lambda_{*}\right) \in \Delta_{I *}$, est tel que $\lambda_{*} \neq 1$, on note $\lambda^{\prime}$ le point de $\Delta_{I}$ défini par 


$$
\lambda_{i_{v}}^{\prime}=\frac{\lambda_{i_{v}}}{1-\lambda_{*}} \quad(v=1, \ldots, l) .
$$

On considère une fonction $\chi_{\varepsilon}$ de classe $\mathcal{C}^{\infty}$ de $[0,1]$ dans lui-même, identiquement nulle au voisinage de 0 et identiquement égale à 1 au voisinage de 1 , qui vérifie de plus $\left|\chi_{\varepsilon}(t)-t\right|<\varepsilon$ pour tout $t \in[0,1]$.

Notons $w^{I}$ et $\Phi_{I}$ les fonctions construites dans la Section 3 pour le multi-indice I. On pose alors pour $\lambda \in \Delta_{I *}$

$$
\begin{aligned}
w^{I *}(z, \zeta, \lambda)= & \left(1-\lambda_{*}\right)\left(2 \frac{\partial \rho_{\lambda^{\prime}}}{\partial \zeta_{j}}(\zeta)-\sum_{k=1}^{n} a_{j k}^{\lambda^{\prime}}(\zeta)\left(\zeta_{k}-z_{k}\right)\right) \\
& +\left(1-\chi_{\varepsilon}\left(\lambda_{*}\right)\right) A \sum_{k=1}^{n} \overline{Q_{j k}^{\lambda^{\prime}}\left(\zeta_{k}-z_{k}\right)} \\
& +\lambda_{*}\left(2 \frac{\partial \rho_{*}}{\partial \zeta_{j}}(\zeta)-\sum_{k=1}^{n} a_{j k}^{*}(\zeta)\left(\zeta_{k}-z_{k}\right)\right) \\
& +\chi_{\varepsilon}\left(\lambda_{*}\right) B \sum_{k=1}^{n} \overline{Q_{j k}^{*}\left(\zeta_{k}-z_{k}\right)} \\
\Phi_{I *}(z, \zeta, \lambda)= & \left\langle w^{I *}(z, \zeta, \lambda), \zeta-z\right\rangle .
\end{aligned}
$$

Notons que $w^{I *}=w^{I}$ et $\Phi_{I *}=\Phi_{I}$, si $\lambda_{*}=0$, et $w^{I *}=w^{*}$ et $\Phi_{I *}=\Phi_{*}$, si $\lambda_{*}=1$.

La fonction $\Phi_{I *}$ s'écrit

$$
\Phi_{I *}(z, \zeta, \lambda)=\tilde{F}_{\lambda}(\zeta, z)+\left\langle P^{\lambda}(\zeta-z), \bar{\zeta}-\bar{z}\right\rangle,
$$

où $P^{\lambda}$ est l'opérateur défini par $\left(1-\chi_{\varepsilon}\left(\lambda_{*}\right)\right) A Q^{\lambda^{\prime}}+\chi_{\varepsilon}\left(\lambda_{*}\right) B Q^{*}$ et si $\varepsilon$ est choisi assez petit et $\gamma=\frac{1}{2} \min \left(\alpha, \alpha^{*}\right)$, où $\alpha$ est la constante de (3.8), on a

$$
\operatorname{Re} \Phi_{I *}(z, \zeta, \lambda) \geq \rho_{\lambda}(\zeta)-\rho_{\lambda}(z)+\frac{\gamma}{2}|\zeta-z|^{2}
$$

On définit des sections de Leray $\left(\psi_{J}\right)_{J \in \mathcal{I}^{\prime}(k, *)}$ au voisinage de $z_{0}$ en posant pour $J=I *$

$$
\psi_{J}(z, \zeta, \lambda)=\frac{w^{I *}(z, \zeta, \lambda)}{\Phi_{I *}(z, \zeta, \lambda)}
$$

Remarquons que $\left.\psi_{J}\right|_{U_{z_{0}} \times U_{z_{0}} \backslash \Delta\left(U_{z_{0}}\right) \times \Delta_{I}}=\psi_{I}$. A ces sections de Leray on associe les noyaux $K_{0 I *}(z, \zeta, \lambda)$ et $K_{I *}(z, \zeta, \lambda)$, pour $(z, \zeta, \lambda) \in U_{z_{0}} \times U_{z_{0}} \backslash \Delta\left(U_{z_{0}}\right) \times \Delta_{0 I *}$, définis par

$$
K_{0 I *}(z, \zeta, \lambda)=\frac{(-1)^{n(n-1) / 2}}{(2 i \pi)^{n}}\left\langle\psi_{0 I *}, d(\zeta-z)\right\rangle \wedge\left\langle\left(\bar{\partial}_{z, \zeta}+d_{\lambda}\right) \psi_{0 I *}, d(\zeta-z)\right\rangle^{n-1}
$$

et par

$$
K_{I *}(z, \zeta, \lambda)=\frac{(-1)^{n(n-1) / 2}}{(2 i \pi)^{n}}\left\langle\psi_{I *}, d(\zeta-z)\right\rangle \wedge\left\langle\left(\bar{\partial}_{z, \zeta}+d_{\lambda}\right) \psi_{I *}, d(\zeta-z)\right\rangle^{n-1} .
$$


On pose également, pour $(z, \zeta) \in U_{z_{0}} \times U_{z_{0}} \backslash \Delta\left(U_{z_{0}}\right)$,

$$
\begin{aligned}
& B_{I *}(z, \zeta)=C_{0 I *}(z, \zeta)=\int_{\lambda \in \Delta_{0 I *}} K_{0 I *}(z, \zeta, \lambda), \\
& C_{I *}(z, \zeta)=\int_{\lambda \in \Delta_{I *}} K_{I *}(z, \zeta, \lambda),
\end{aligned}
$$

et comme précédemment on a

$$
\bar{\partial}_{z, \zeta} B_{I *}=B_{\delta(I *)}-C_{I *} .
$$

Lemme 4.1. Pour $(z, \zeta) \in U_{z_{0}} \times U_{z_{0}} \backslash \Delta\left(U_{z_{0}}\right)$, on a la relation

$$
\bar{\partial}_{z, \zeta}\left(\sum_{I \in \mathcal{I}^{\prime}(k)} \operatorname{sgn}(I) B_{I *}\right)=(-1)^{k} \sum_{I \in \mathcal{I}^{\prime}(k)} \operatorname{sgn}(I) B_{I}-\sum_{I \in \mathcal{I}^{\prime}(k)} \operatorname{sgn}(I) C_{I *} .
$$

Démonstration. Grâce à la relation (4.5) on a

$$
\bar{\partial}_{z, \zeta} B_{I *}=(-1)^{k} B_{I}-C_{I *}+\sum_{\nu=1}^{k}(-1)^{\nu+1} B_{I(\hat{v}) *} .
$$

Comme on l'a déjà remarqué dans la démonstration du Lemme 2.6

$$
\sum_{I \in \mathcal{I}^{\prime}(k)} \operatorname{sgn}(I) \sum_{\nu=1}^{k}(-1)^{v+1} B_{I(\hat{v}) *}=0
$$

ce qui prouve la relation (4.6).

ThÉorème 4.2. Le noyau $R_{M}=\sum_{I \in \mathcal{I}^{\prime}(k)} \operatorname{sgn}(I) C_{I *}$ est une solution fondamentale de l'opérateur de Cauchy-Riemann tangentiel au voisinage de $z_{0}$. Plus précisément, on a pour $0 \leq p \leq n$ et $n-k-q+1 \leq r \leq n-k$

$$
\bar{\partial}_{z}\left[R_{M}\right]_{p, r-1}+\bar{\partial}_{\zeta}\left[R_{M}\right]_{p, r}=(-1)^{k(k+3) / 2}\left[\Delta\left(U_{z 0}\right)\right],
$$

où $\left[R_{M}\right]_{p, r}$ désigne la composante de bidegré $(p, r)$ en $z$ de $R_{M}$.

Démonstration. Puisque $B_{M}=\sum_{I \in \mathcal{I}^{\prime}(k)} \operatorname{sgn}(I) B_{I}$ satisfait la relation (4.1), il suffit de prouver que

$$
\bar{\partial}_{z, \zeta}\left(\sum_{I \in \mathcal{I}^{\prime}(k)} \operatorname{sgn}(I) B_{I *}\right)=(-1)^{k} B_{M}-R_{M}
$$

au sens des courants sur $U_{z_{0}} \times U_{z_{0}}$.

Soit $\chi$ une fonction de classe $\mathcal{C}^{\infty}$ sur $\mathbb{R}$ à valeurs dans $[0,1]$ telle que $\chi(x)=1$ si $|x| \leq 1 / 2$ et $\chi(x)=0$ si $|x| \geq 1$. On pose $\chi_{\varepsilon}(z, \zeta)=\chi(|\zeta-z| / \varepsilon)$. Notons pour simplifier les écritures $E_{M}=\sum_{I \in \mathcal{I}^{\prime}(k)} \operatorname{sgn}(I) B_{I *}$.

Puisque $1-\chi_{\varepsilon}$ est identiquement nulle au voisinage de la diagonale de $U_{z_{0}} \times U_{z_{0}}$, il résulte du Lemme 4.1 que

$$
\bar{\partial}_{z, \zeta}\left(\left(1-\chi_{\varepsilon}\right) E_{M}\right)=\bar{\partial}_{z, \zeta}\left(1-\chi_{\varepsilon}\right) \wedge E_{M}+\left(1-\chi_{\varepsilon}\right)\left((-1)^{k} B_{M}-R_{M}\right)
$$

au sens des courants sur $U_{z_{0}} \times U_{z_{0}}$. 
Le Lemme 3.8 nous permet d' affirmer que $\left(1-\chi_{\varepsilon}\right) B_{M}$ converge vers $B_{M}$ faiblement au sens des courants sur $U_{z_{0}} \times U_{z_{0}}$ quand $\varepsilon$ tend vers 0 . Il nous reste à montrer que $\left(1-\chi_{\varepsilon}\right) R_{M},\left(1-\chi_{\varepsilon}\right) E_{M}$, et $\bar{\partial}_{z, \zeta}\left(\left(1-\chi_{\varepsilon}\right) E_{M}\right)$ convergent respectivement vers $R_{M}, E_{M}$ et $\bar{\partial}_{z, \zeta} E_{M}$ faiblement au sens des courants sur $U_{z_{0}} \times U_{z_{0}}$ quand $\varepsilon$ tend vers 0 et que $\bar{\partial}_{z, \zeta}\left(1-\chi_{\varepsilon}\right) \wedge E_{M}$ converge faiblement vers 0 .

Soit $f$ une forme différentielle de classe $\mathcal{C}^{\infty}$ à support compact dans $U_{z_{0}}$. On a

$$
\begin{aligned}
\int_{\zeta \in M} & \left(1-\chi_{\varepsilon}(z, \zeta)\right) f(\zeta) \wedge E_{M}(z, \zeta) \\
= & \sum_{I \in \mathcal{I}^{\prime}(k)} \operatorname{sgn}(I) \int_{\zeta \in M}\left(1-\chi_{\varepsilon}(z, \zeta)\right) f(\zeta) \wedge B_{I *}(z, \zeta) \\
= & \sum_{I \in \mathcal{I}^{\prime}(k)} \operatorname{sgn}(I) \int_{(\zeta, \lambda) \in M \times \Delta_{0 I *}}\left(1-\chi_{\varepsilon}(z, \zeta)\right) f(\zeta) \wedge K_{0 I *}(z, \zeta, \lambda)
\end{aligned}
$$

Par définition des noyaux $K_{0 I *}$, des estimations analogues à celles effectuées dans la Section 3 pour le noyau $K_{0 I}$ nous donnent

$$
\begin{aligned}
& {\left[f(\zeta) \wedge K_{0 I *}(z, \zeta, \lambda)\right]_{\operatorname{deg} \lambda=|I|+1}} \\
& \quad=\sum_{\substack{0 \leq s \leq n-2-|I| \\
0 \leq m \leq|I|}} \frac{O_{|I|-2 n+2(|I|+1+s-m)+m+2}}{\Phi^{(|I|+1+s-m)+m}} \wedge \partial \rho_{i_{1}}(\zeta) \wedge \cdots \wedge \partial \rho_{i_{m}}(\zeta) .
\end{aligned}
$$

En bornant $m$ par $|I|=k$, nous avons tenu compte du fait que si $I=\left(i_{1}, \ldots, i_{k}\right) \in$ $\mathcal{I}^{\prime}(k)$ on a $\partial \rho_{i_{1}}(\zeta) \wedge \cdots \wedge \partial \rho_{i_{k}}(\zeta) \wedge \partial \rho_{*}(\zeta)=0$ car $\rho_{*}$ est une combinaison linéaire de $\rho_{1}, \ldots, \rho_{k}$.

Soient $\sigma$ un monôme en $d \zeta_{1}, \ldots, d \zeta_{n}, d \bar{\zeta}_{1}, \ldots, d \bar{\zeta}_{n}, \lambda^{1}, \ldots, \lambda^{k+1}$ des points de $\Delta_{I *}$ qui forment un système de vecteurs indépendants de $\mathbb{R}^{k+1}, t_{v}=\operatorname{Im} \Phi\left(z, \zeta, \lambda^{v}\right)$ et $d t_{v}=d_{\zeta} \operatorname{Im} \Phi\left(z, \zeta, \lambda^{\nu}\right)$. Par définition de $\Phi$, on a

$$
d t_{v}(z, \zeta)=i\left(\bar{\partial} \rho_{\lambda^{v}}(\zeta)-\partial \rho_{\lambda^{v}}(\zeta)\right)+O_{1}
$$

et par conséquent

$$
\partial \rho_{\lambda^{v}}(\zeta)=\frac{1}{2} d \rho_{\lambda^{v}}(\zeta)+\frac{i}{2} d t_{v}(z, \zeta)+O_{1}
$$

Comme $\left.d \rho_{\lambda^{\nu}}\right|_{M}=0$ pour tout $1 \leq v \leq k+1$, il existe une constante $C$ et des monômes $\sigma_{L}$ en $d \zeta_{1}, \ldots, d \zeta_{n}, d \bar{\zeta}_{1}, \ldots, d \bar{\zeta}_{n}$ tels que pour tout $i_{1}, \ldots, i_{m} \in I *$

$$
\left|\left(\sigma \wedge \partial \rho_{i_{1}}(\zeta) \wedge \cdots \wedge \partial \rho_{i_{m}}\right)\right|_{M}\left|\leq C \sum_{|L| \leq m}\right| \sigma_{L} \wedge_{l \in L} d t_{l}|| \zeta-\left.z\right|^{m-|L|}
$$

Il résulte alors du paragraphe 6 et du Lemme 7.4 de [16] et de l'inégalité (4.2) qu'après intégration partielle en $\lambda,\left|\int_{\zeta \in M} \chi_{\varepsilon}(z, \zeta) f(\zeta) \wedge E_{M}(z, \zeta)\right|$ est contrôlé par une somme finie d'intégrales de la forme:

$$
M_{s, \varepsilon}=\int_{\zeta \in M \cap B(z, \varepsilon)} \frac{\left|\sigma_{s} \bigwedge_{\nu=1}^{s} d t_{\nu}\right|}{\prod_{\nu=1}^{s}\left(\left|t_{\nu}\right|+|\zeta-z|^{2}\right)|\zeta-z|^{2 n-k-s-2}},
$$

où $1 \leq s \leq k$. 
Il nous reste à prouver que les fonctions de toute famille à $k$ éléments extraite de la famille $\left(t_{v}\right)_{1 \leq v \leq k+1}$ peuvent être utilisée comme coordonnées locales. Pour tout $v, 1 \leq v \leq k+1$, on a $\partial \rho_{\lambda^{v}}=\frac{i}{2} d t_{v}+O_{1}$ sur $M$, il suffit donc de montrer que $\partial \rho_{\lambda^{\nu_{1}}} \wedge \cdots \wedge \partial \rho_{\lambda^{\nu_{k}}} \neq 0$, pour tout $k$-uplet $\left(v_{1}, \ldots, v_{k}\right)$ extrait de $(1, \ldots, k+1)$.

Puisque $\rho_{\lambda}$ dépend linéairement de $\lambda$, l'indépendance linéaire des vecteurs $\lambda^{1}, \ldots, \lambda^{k+1}$ implique l'existence d'une matrice $A$ inversible d'ordre $k+1$ telle que $\partial \rho_{\lambda} \bullet=A \partial \rho_{I *}$, où $\partial \rho_{\lambda}$ • est le vecteur colonne dont les coordonnées sont les $\partial \rho_{\lambda^{\nu}}, v=1, \ldots, k+1$, et $\partial \rho_{I *}$ le vecteur colonne de coordonnées $\partial \rho_{i_{1}}, \ldots, \partial \rho_{i_{k}}, \partial \rho_{*}$, si $I=\left(i_{1}, \ldots, i_{k}\right)$.

Si $\rho_{*}=\rho_{1}+\cdots+\rho_{k}$, on a, par définition des $\rho_{i_{j}},\left|i_{j}\right|=j, \partial \rho_{*}=\varepsilon_{1} \partial \rho_{i_{1}}+$ $\cdots+\varepsilon_{k} \partial \rho_{i_{k}}$, où $\varepsilon_{j}=i_{j} /\left|i_{j}\right|$. On note $L_{k}$ la matrice carrée d'ordre $k+1$ dont les $k$ premières lignes sont identiquement nulles et la $k+$ lième ligne vaut $\left(\varepsilon_{1} \ldots \varepsilon_{k}-1\right)$ et $B$ la matrice rectangulaire $k+1 \times k$, qui coïncide avec la matrice $A+A L_{k}$ privée de sa $(k+1)$ ième colonne identiquement nulle. On a $\partial \rho_{\lambda} \bullet=B \partial \rho_{I}$. De plus toute matrice carrée $B_{k}$ d'ordre $k$ extraite de $B$ est inversible et donc

$$
\partial \rho_{\lambda^{v_{1}}} \wedge \cdots \wedge \partial \rho_{\lambda^{v_{k}}}=\operatorname{det}\left(B_{k}\right) \partial \rho_{i_{1}} \wedge \cdots \wedge \partial \rho_{i_{k}} \neq 0,
$$

car $M$ est générique.

On peut maintenant intégrer en utilisant les coordonnées $t_{v}$ et on obtient

$$
\left|\int_{\zeta \in M} \chi_{\varepsilon}(z, \zeta) f(\zeta) \wedge E_{M}(z, \zeta)\right| \lesssim \varepsilon^{2}(1+|\ln \varepsilon|)^{k} .
$$

Les mêmes estimations, associées au fait que $\left|\bar{\partial}_{z, \zeta}\left(1-\chi_{\varepsilon}\right)\right| \leq C_{1} / \varepsilon$, impliquent que

$$
\left|\int_{\zeta \in M} \bar{\partial}_{z, \zeta}\left(1-\chi_{\varepsilon}(z, \zeta)\right) f(\zeta) \wedge E_{M}(z, \zeta)\right| \lesssim \varepsilon(1+|\ln \varepsilon|)^{k} .
$$

Nous avons donc prouvé que $\left(1-\chi_{\varepsilon}\right) E_{M}$ converge faiblement vers $E_{M}$ et que $\bar{\partial}_{z, \zeta}\left(1-\chi_{\varepsilon}\right) \wedge E_{M}$ converge faiblement vers 0 quand $\varepsilon$ tend vers 0 . De plus l'opérateur $\bar{\partial}_{z, \zeta}$ étant faiblement continu, $\bar{\partial}_{z, \zeta}\left(\left(1-\chi_{\varepsilon}\right) E_{M}\right)$ converge faiblement vers $\bar{\partial}_{z, \zeta} E_{M}$.

Etudions maintenant la convergence de $\left(1-\chi_{\varepsilon}\right) R_{M}$. Soit $f$ une forme différentielle de classe $\mathcal{C}^{\infty}$ à support compact dans $U_{z_{0}}$. On a

$$
\begin{aligned}
\int_{\zeta \in M} & \left(1-\chi_{\varepsilon}(z, \zeta)\right) f(\zeta) \wedge R_{M}(z, \zeta) \\
& =\sum_{I \in \mathcal{I}^{\prime}(k)} \operatorname{sgn}(I) \int_{\zeta \in M}\left(1-\chi_{\varepsilon}(z, \zeta)\right) f(\zeta) \wedge C_{I *}(z, \zeta) \\
& =\sum_{I \in \mathcal{I}^{\prime}(k)} \operatorname{sgn}(I) \int_{(\zeta, \lambda) \in M \times \Delta_{I *}}\left(1-\chi_{\varepsilon}(z, \zeta)\right) f(\zeta) \wedge K_{I *}(z, \zeta, \lambda) .
\end{aligned}
$$

Par définition (cf. (4.4)), des noyaux $K_{I *}$ on a

$$
\begin{aligned}
f(\zeta) \wedge K_{I *}(z, \zeta, \lambda)= & \frac{(-1)^{n(n-1) / 2}}{(2 i \pi)^{n}} \tilde{f}(\zeta) \wedge\left\langle\psi_{I *}, d \zeta\right\rangle \\
& \wedge\left\langle\left(\bar{\partial}_{z, \zeta}+d_{\lambda}\right) \psi_{I *}, d \zeta\right\rangle^{n-1} \wedge d z_{1} \wedge \cdots \wedge d z_{n}
\end{aligned}
$$


Comme on doit intégrer $f(\zeta) \wedge K_{I *}(z, \zeta, \lambda)$ sur $\Delta_{I *}$, qui est de dimension réelle $|I|$, seule la composante de degré $|I|$ en $\lambda$ du noyau $K_{I *}$ donnera une contribution. Par des calculs analogues à ceux de la Section 3, on obtient

$$
\begin{aligned}
{\left[f(\zeta) \wedge K_{I *}(z, \zeta, \lambda)\right]_{\operatorname{deg} \lambda=|I|} } & =\frac{O_{0} \wedge W}{\Phi^{n}} \wedge\left(d_{\lambda} W\right)^{|I|} \\
& =\sum_{\substack{0 \leq m \leq|I| \\
i_{1}, \ldots, i_{m} \in I *}} \frac{O_{|I|+1-m}}{\Phi^{n}} \wedge \partial \rho_{i_{1}}(\zeta) \wedge \cdots \wedge \partial \rho_{i_{m}}(\zeta)
\end{aligned}
$$

Notons que comme dans le cas précédent $m \leq|I|=k$ et remarquons que, puisque la variété $M$ n'est pas totalement réelle, on a $n>k$. Avec les notations ci-dessus, après intégration partielle en $\lambda,\left|\int_{\zeta \in M} \chi_{\varepsilon}(z, \zeta) f(\zeta) \wedge R_{M}(z, \zeta)\right|$ est contrôlé, grâce à (4.2), par une somme finie d'intégrales de la forme:

$$
N_{s, \varepsilon}=\int_{\zeta \in M \cap B(z, \varepsilon)} \frac{\left|\sigma_{s} \bigwedge_{\nu=1}^{s} d t_{\nu}\right|}{\prod_{\nu=1}^{s}\left(\left|t_{\nu}\right|+|\zeta-z|^{2}\right)|\zeta-z|^{2 n-k-s-1}},
$$

où $1 \leq s \leq k$.

En utilisant de nouveau les $t_{v}$ comme coordonnées locales, on obtient donc que

$$
\left|\int_{\zeta \in M} \chi_{\varepsilon}(z, \zeta) f(\zeta) \wedge R_{M}(z, \zeta)\right| \lesssim \varepsilon(1+|\ln \varepsilon|)^{k} .
$$

Nous avons donc prouvé que $\left(1-\chi_{\varepsilon}\right) R_{M}$ converge faiblement vers $R_{M}$.

Corollaire 4.3. Soit $\Omega$ un domaine à bord $\mathcal{C}^{1}$ relativement compact dans $M \cap$ $U_{z_{0}}$.

Si f est une $(n, r)$-forme de classe $\mathcal{C}^{1}$ dans $\bar{\Omega}, n-k-q+1 \leq r \leq n-k$, alors on a la formule suivante au sens des courants sur $M$

$$
\begin{aligned}
(-1)^{(k+1)(r+n)+\frac{k(k+1)}{2}} f(z)= & \bar{\partial}_{b} \int_{\zeta \in \Omega} f(\zeta) \wedge R_{M}(z, \zeta) \\
& +(-1)^{k+1} \int_{\zeta \in \Omega} \bar{\partial}_{b} f(\zeta) \wedge R_{M}(z, \zeta) \\
& +(-1)^{k} \int_{\zeta \in \partial \Omega} f(\zeta) \wedge R_{M}(z, \zeta)
\end{aligned}
$$

Si $f$ est une $(n, r)$-forme de classe $\mathcal{C}^{1}$ dans $\bar{\Omega}, 0 \leq r \leq q-1$, alors on a la formule suivante au sens des courants sur $M$

$$
\begin{aligned}
(-1)^{(k+1)(r+n)+\frac{k(k+1)}{2}} f(\zeta)= & \bar{\partial}_{b} \int_{z \in \Omega} f(z) \wedge R_{M}(z, \zeta) \\
& +(-1)^{k+1} \int_{z \in \Omega} \bar{\partial}_{b} f(z) \wedge R_{M}(z, \zeta) \\
& +(-1)^{k} \int_{z \in \partial \Omega} f(z) \wedge R_{M}(z, \zeta)
\end{aligned}
$$




\section{Estimations}

On désigne toujours par $M$ une sous-variété CR générique, $q$-concave, $q \geq 1$, de codimension réelle $k$ dans $\mathbb{C}^{n}$ et par $z_{0}$ un point de $M$. Dans la Section 4 nous avons défini une solution fondamentale $R_{M}$ de l'opérateur de Cauchy-Riemann tangentiel sur $M$ dans un voisinage $U_{z_{0}}$ de $z_{0}$. Soit $\Omega$ un domaine relativement compact dans $M \cap U_{z_{0}}$. Pour toute $(n, r)$-forme $f$ à coefficients dans $L^{\infty}(\Omega)$, on pose

$$
\begin{aligned}
& \tilde{R}_{M} f(z)=\int_{\zeta \in \Omega} f(\zeta) \wedge R_{M}(z, \zeta) \quad \text { si } n-k-q+1 \leq r \leq n-k, \\
& \tilde{R}_{M} f(\zeta)=\int_{z \in \Omega} f(z) \wedge R_{M}(z, \zeta) \quad \text { si } 0 \leq r \leq q .
\end{aligned}
$$

Nous allons étudier les propriétés de régularité de l'opérateur $\tilde{R}_{M}$ en fonction de la régularité de la variété $M$.

\subsection{Estimations Hölderiennes}

On note $W_{n, r}^{0, \infty}(\Omega)$ l'espace des $(n, r)$-formes différentielles à coefficients dans $L^{\infty}(\Omega)$ et $\mathcal{C}_{n, r}^{1 / 2}(\Omega)$ l'espace des $(n, r)$-formes différentielles à coefficients hölderiens d'ordre $1 / 2$.

THÉORÈme 5.1. Si $M$ est de classe $\mathcal{C}^{2}$ et $n-k-q+1 \leq r \leq n-k$, l'opérateur $\tilde{R}_{M}$ est continu de $W_{n, r}^{0, \infty}(\Omega)$ dans $\mathcal{C}_{n, r-1}^{1 / 2}(\Omega)$.

Démonstration. Soient $z_{1}$ et $z_{2}$ des points de $\Omega$. On a

et par conséquent

$$
\tilde{R}_{M} f\left(z_{1}\right)-\tilde{R}_{M} f\left(z_{2}\right)=\int_{\zeta \in \Omega} f(\zeta) \wedge\left(R_{M}\left(z_{1}, \zeta\right)-R_{M}\left(z_{2}, \zeta\right)\right),
$$

$$
\begin{aligned}
& \left|\tilde{R}_{M} f\left(z_{1}\right)-\tilde{R}_{M} f\left(z_{2}\right)\right| \leq \int_{\substack{\zeta \in \Omega \\
\left|\zeta-z_{1}\right| \leq 2\left|z_{1}-z_{2}\right|^{1 / 2}}}\left|f(\zeta) \wedge\left(R_{M}\left(z_{1}, \zeta\right)-R_{M}\left(z_{2}, \zeta\right)\right)\right| \\
& +\int_{\substack{\zeta \in \Omega \\
\left|\zeta-z_{1}\right| \geq 2\left|z_{1}-z_{2}\right|^{1 / 2}}}\left|f(\zeta) \wedge\left(R_{M}\left(z_{1}, \zeta\right)-R_{M}\left(z_{2}, \zeta\right)\right)\right| .
\end{aligned}
$$

L'opérateur $R_{M}$ étant linéaire, on peut supposer sans perte de généralité que $f$ est de la forme $f=\tilde{f} \sigma$, où $\tilde{f}$ est une fonction dans $L^{\infty}(\Omega)$ et $\sigma$ un monôme en $d \zeta_{1}, \ldots, d \zeta_{n}, d \bar{\zeta}_{1}, \ldots, d \bar{\zeta}_{n}$. On obtient alors

$$
\begin{aligned}
\left|\tilde{R}_{M} f\left(z_{1}\right)-\tilde{R}_{M} f\left(z_{2}\right)\right| & |\sigma| \|_{\infty} \int_{\substack{\zeta \in \Omega \\
\left|\zeta-z_{1}\right| \leq 2\left|z_{1}-z_{2}\right|^{1 / 2}}}\left|\sigma \wedge\left(R_{M}\left(z_{1}, \zeta\right)-R_{M}\left(z_{2}, \zeta\right)\right)\right| \\
& +\|f\|_{\infty} \int_{\substack{\zeta \in \Omega \\
\left|\zeta-z_{1}\right| \geq 2\left|z_{1}-z_{2}\right|^{1 / 2}}}\left|\sigma \wedge\left(R_{M}\left(z_{1}, \zeta\right)-R_{M}\left(z_{2}, \zeta\right)\right)\right| .
\end{aligned}
$$


Nous devons donc estimer les intégrales

$$
\begin{aligned}
& J_{1}=\int_{\substack{\zeta \in \Omega \\
\left|\zeta-z_{1}\right| \leq 2\left|z_{1}-z_{2}\right|^{1 / 2}}}\left|\sigma \wedge\left(R_{M}\left(z_{1}, \zeta\right)-R_{M}\left(z_{2}, \zeta\right)\right)\right|, \\
& J_{2}=\int_{\substack{\zeta \in \Omega \\
\left|\zeta-z_{1}\right| \geq 2\left|z_{1}-z_{2}\right|^{1 / 2}}}\left|\sigma \wedge\left(R_{M}\left(z_{1}, \zeta\right)-R_{M}\left(z_{2}, \zeta\right)\right)\right| .
\end{aligned}
$$

Nous supposerons que $\left|z_{1}-z_{2}\right| \leq 1$. Remarquons que

$$
J_{1} \leq \int_{\substack{\zeta \in \Omega \\\left|\zeta-z_{1}\right| \leq 2\left|z_{1}-z_{2}\right|^{1 / 2}}}\left|\sigma \wedge R_{M}\left(z_{1}, \zeta\right)\right|+\int_{\substack{\zeta \in \Omega \\\left|\zeta-z_{2}\right| \leq 3\left|z_{1}-z_{2}\right|^{1 / 2}}}\left|\sigma \wedge R_{M}\left(z_{2}, \zeta\right)\right| .
$$

Nous avons vu dans le paragraphe précédent que

$$
\begin{aligned}
\sigma \wedge R_{M}(z, \zeta) & =\sum_{I \in \mathcal{I}^{\prime}(k)} \operatorname{sgn}(I) \int_{\lambda \in \Delta_{I *}}\left[\sigma \wedge K_{I *}(z, \zeta, \lambda)\right]_{\operatorname{deg} \lambda=|I|} \\
& =\sum_{I \in \mathcal{I}^{\prime}(k)} \sum_{\substack{0 \leq m \leq k \\
i_{1}, \ldots, i_{m} \in I *}} \int_{\lambda \in \Delta_{I *}} \frac{O_{|I|+1-m}}{\Phi^{n}} \wedge \partial \rho_{i_{1}}(\zeta) \wedge \cdots \wedge \partial \rho_{i_{m}}(\zeta)
\end{aligned}
$$

La variété $M$ est supposée $q$-concave avec $q \geq 1$ et par conséquent $n>k+1$. L'intégration partielle par rapport à $\lambda$ permet de contrôler

$$
\int_{\zeta \in \Omega}\left|\sigma \wedge R_{M}\left(z_{j}, \zeta\right)\right|, \quad j=1,2,
$$

par une somme finie d'intégrales de la forme:

$$
A\left(z_{1}, z_{2}\right)=\int_{\substack{\zeta \in \Omega \\|\zeta-z| \leq c_{j}\left|z_{1}-z_{2}\right|^{1 / 2}}} \frac{\left|\sigma \wedge \partial \rho_{i_{1}}(\zeta) \wedge \cdots \wedge \partial \rho_{i_{m}}(\zeta)\right|}{\prod_{\nu=1}^{k+1}\left|\Phi\left(z, \zeta, \lambda^{\nu}\right)\right||\zeta-z|^{2 n-3 k+m-3}},
$$

où $\lambda^{1}, \ldots, \lambda^{k+1}$ sont des points de $\Delta_{I *}, I \in \mathcal{I}^{\prime}(k)$, qui forment un système de vecteurs indépendants de $\mathbb{R}^{k+1}$.

On pose toujours $t_{v}=\operatorname{Im} \Phi\left(z, \zeta, \lambda^{\nu}\right)$ et $d t_{v}=d_{\zeta} \operatorname{Im} \Phi\left(z, \zeta, \lambda^{v}\right)$. Il résulte de la définition de $\rho_{*}$ et de l'étude faite dans la Section 4 que pour tout multi-indice $\left(v_{1}, \ldots, v_{k}\right)$ extrait de $(1, \ldots, k+1)$, on a

$$
\left|\sigma \wedge \partial \rho_{i_{1}}(\zeta) \wedge \cdots \wedge \partial \rho_{i_{m}}\right| \lesssim \sum_{0 \leq|L| \leq m}\left|\sigma_{L} \wedge_{l \in L} d t_{l}\right||\zeta-z|^{m-|L|}
$$

où $L=\left(l_{1}, \ldots, l_{|L|}\right)$ est un multi-indice de longueur $|L| \leq k$ extrait de $\left(v_{1}, \ldots, v_{k}\right)$.

Comme $|L| \leq k$, il existe $v_{L} \in\{1, \ldots, k+1\} \backslash L$ et d'après (4.2)

$$
\begin{aligned}
& \prod_{\nu=1}^{k+1}\left|\Phi\left(z, \zeta, \lambda^{\nu}\right)\right||\zeta-z|^{2 n-3 k+|L|-3} \\
& \quad \geq \prod_{l \in L}\left|\Phi\left(z, \zeta, \lambda^{l}\right)\right|\left|\Phi\left(z, \zeta, \lambda^{\nu_{L}}\right)\right||\zeta-z|^{2 n-k-|L|-3} .
\end{aligned}
$$


Le terme de $A\left(z_{1}, z_{2}\right)$ correspondant à un multi-indice $L=\emptyset$ est majoré par

$$
\begin{aligned}
A_{0}\left(z_{1}, z_{2}\right) & \lesssim \int_{\substack{X \in \mathbb{R}^{2 n-k} \\
|X| \leq c_{j}\left|z_{1}-z_{2}\right|^{1 / 2}}} \frac{d X}{|X|^{2 n-k-1}} \\
& \lesssim\left|z_{1}-z_{2}\right|^{1 / 2} .
\end{aligned}
$$

Si $0<|L|<k$, les fonctions $t_{l_{1}}, \ldots, t_{l_{|L|}}, t_{v_{L}}$ peuvent être utilisées comme coordonnées locales ainsi que nous l'avons remarqué dans la Section 4. Les termes de $A\left(z_{1}, z_{2}\right)$ correspondant à un multi-indice $L$ de longueur strictement inférieure à $k$ sont donc contrôlés par:

$$
\begin{aligned}
A_{L}\left(z_{1}, z_{2}\right) & \lesssim \int_{\substack{X \in \mathbb{R}^{2 n-k} \\
|X| \leq c_{j}\left|z_{1}-z_{2}\right|^{1 / 2}}} \frac{d X}{\prod_{\nu=1}^{|L|+1}\left(\left|X_{\nu}\right|+|X|^{2}\right)|X|^{2 n-k-|L|-3}} \\
& \lesssim\left|z_{1}-z_{2}\right|\left(1+|\ln | z_{1}-z_{2}||\right)^{|L|+1}
\end{aligned}
$$

$\mathrm{Si}|L|=k$, on a

$$
\prod_{l \in L}\left|\Phi\left(z, \zeta, \lambda^{l}\right)\right|\left|\Phi\left(z, \zeta, \lambda^{\nu_{L}}\right)\right|=\prod_{\nu=1}^{k+1}\left|\Phi\left(z, \zeta, \lambda^{\nu}\right)\right| .
$$

En remarquant que

$$
\prod_{\nu=1}^{k+1}\left|\Phi\left(z, \zeta, \lambda^{\nu}\right)\right| \geq \min _{\nu_{1}, \ldots, \nu_{k} \in\{1, \ldots, k+1\}} \prod_{j=1}^{k}\left|\Phi\left(z, \zeta, \lambda^{\nu_{j}}\right)\right|^{1+1 / k},
$$

on peut majorer le terme de $A\left(z_{1}, z_{2}\right)$ correspondant à $L$ de longueur $k$ de la manière suivante:

$$
\begin{aligned}
A_{L}\left(z_{1}, z_{2}\right) & \lesssim \int_{\substack{X \in \mathbb{R}^{2 n-k} \\
|X| \leq c_{j}\left|z_{1}-z_{2}\right|^{1 / 2}}} \frac{d X}{\prod_{\nu=1}^{k}\left(\left|X_{v}\right|+|X|^{2}\right)^{1+1 / k}|X|^{2 n-2 k-3}} \\
& \lesssim\left|z_{1}-z_{2}\right|^{1 / 2} .
\end{aligned}
$$

On en déduit donc que $J_{1} \lesssim\left|z_{1}-z_{2}\right|^{1 / 2}$. Nous allons maintenant étudier $J_{2}$. Il résulte de la définition de $J_{2}$ et de (5.3) que

$$
\begin{aligned}
& J_{2}=\sum_{I \in \mathcal{I}^{\prime}(k)} \int_{\substack{\zeta, \lambda) \in \Omega \times \Delta_{I *} \\
\left|\zeta-z_{1}\right| \geq 2\left|z_{1}-z_{2}\right|^{1 / 2}}} \mid \frac{N\left(z_{1}, \zeta, \lambda\right)}{\Phi^{n}\left(z_{1}, \zeta, \lambda\right)} \\
&-\frac{N\left(z_{2}, \zeta, \lambda\right)}{\Phi^{n}\left(z_{2}, \zeta, \lambda\right)}|| \sigma \wedge \partial \rho_{i_{1}}(\zeta) \wedge \cdots \wedge \partial \rho_{i_{m}}(\zeta) \mid,
\end{aligned}
$$

où $N(z, \zeta, \lambda)$ est une fonction de classe $\mathcal{C}^{\infty}$ en $z$ qui est un $O_{k+1-m}$.

On peut écrire

$$
\begin{aligned}
\frac{N\left(z_{1}, \zeta, \lambda\right)}{\Phi^{n}\left(z_{1}, \zeta, \lambda\right)}-\frac{N\left(z_{2}, \zeta, \lambda\right)}{\Phi^{n}\left(z_{2}, \zeta, \lambda\right)}= & \frac{N\left(z_{1}, \zeta, \lambda\right)-N\left(z_{2}, \zeta, \lambda\right)}{\Phi^{n}\left(z_{1}, \zeta, \lambda\right)} \\
& +N\left(z_{2}, \zeta, \lambda\right)\left[\frac{1}{\Phi^{n}\left(z_{1}, \zeta, \lambda\right)}-\frac{1}{\Phi^{n}\left(z_{2}, \zeta, \lambda\right)}\right]
\end{aligned}
$$


On obtient alors

$$
\begin{aligned}
J_{2}^{\prime} & =\int_{\substack{(\zeta, \lambda) \in \Omega \times \Delta_{I *} \\
\left|\zeta-z_{1}\right| \geq 2\left|z_{1}-z_{2}\right|^{1 / 2}}}\left|\frac{N\left(z_{1}, \zeta, \lambda\right)-N\left(z_{2}, \zeta, \lambda\right)}{\Phi^{n}\left(z_{1}, \zeta, \lambda\right)}\right| \\
& \lesssim\left|z_{1}-z_{2}\right| \sum_{\substack { 0 \leq s \leq k \\
\begin{subarray}{c}{X \in \mathbb{R}^{2 n-k} \\
2\left|z_{1}-z_{2}\right|^{1 / 2} \leq|X| \leq C{ 0 \leq s \leq k \\
\begin{subarray} { c } { X \in \mathbb { R } ^ { 2 n - k } \\
2 | z _ { 1 } - z _ { 2 } | ^ { 1 / 2 } \leq | X | \leq C } }\end{subarray}} \frac{d X}{\prod_{\nu=1}^{s+1}\left(\left|X_{\nu}\right|+|X|^{2}\right)|X|^{2 n-k-s-2}} \\
& \lesssim\left|z_{1}-z_{2}\right|\left(1+|\ln | z_{1}-z_{2}||\right)^{k+1},
\end{aligned}
$$

$\operatorname{car}\left|N\left(z_{1}, \zeta, \lambda\right)-N\left(z_{2}, \zeta, \lambda\right)\right| \leq\left|z_{1}-z_{2}\right| O_{k-m}$.

La fonction $\Phi(z, \zeta, \lambda)$ est de classe $\mathcal{C}^{\infty}$ en $z$ et par conséquent

$$
\frac{1}{\Phi^{n}\left(z_{1}, \zeta, \lambda\right)}-\frac{1}{\Phi^{n}\left(z_{2}, \zeta, \lambda\right)} \lesssim \sum_{p=0}^{n-1} \frac{\left|z_{1}-z_{2}\right|}{\Phi^{n-p}\left(z_{1}, \zeta, \lambda\right) \Phi^{p+1}\left(z_{2}, \zeta, \lambda\right)}
$$

En remarquant que si $\left|\zeta-z_{1}\right| \geq 2\left|z_{1}-z_{2}\right|^{1 / 2}$, alors $1 / 2 \leq\left|\zeta-z_{1}\right| /\left|\zeta-z_{2}\right| \leq 2$, après intégration partielle en $\lambda$ nous obtenons

$$
\begin{aligned}
J_{2}^{\prime \prime}= & \int_{\substack{(\zeta, \lambda) \in \Omega \times \Delta_{I *} \\
\left|\zeta-z_{1}\right| \geq 2\left|z_{1}-z_{2}\right|^{1 / 2}}} N\left(z_{2}, \zeta, \lambda\right)\left|\frac{1}{\Phi^{n}\left(z_{1}, \zeta, \lambda\right)}-\frac{1}{\Phi^{n}\left(z_{2}, \zeta, \lambda\right)}\right| \\
\leq & \left|z_{1}-z_{2}\right| \sum_{1 \leq s \leq k} \int_{\substack{X \in \mathbb{R}^{2 n-k} \\
2\left|z_{1}-z_{2}\right|^{1 / 2} \leq|X| \leq C}} \frac{d X}{\prod_{\nu=1}^{s}\left(\left|X_{\nu}\right|+|X|^{2}\right)^{1+1 / s}|X|^{2 n-k-s-1}} \\
& +\left|z_{1}-z_{2}\right| \int_{\substack{X \in \mathbb{R}^{2 n-k} \\
2\left|z_{1}-z_{2}\right|^{1 / 2} \leq|X| \leq C}} \frac{d X}{\left(\left|X_{1}\right|+|X|^{2}\right)|X|^{2 n-k-1}} \\
& \lesssim\left|z_{1}-z_{2}\right|^{1 / 2}
\end{aligned}
$$

et par conséquent $J_{2} \lesssim\left|z_{1}-z_{2}\right|^{1 / 2}$.

On en déduit donc qu'il existe une constante $C$ telle que si $z_{1}, z_{2} \in \Omega$

$$
\left|\tilde{R}_{M} f\left(z_{1}\right)-\tilde{R}_{M} f\left(z_{2}\right)\right| \leq C\|f\|_{\infty}\left|z_{1}-z_{2}\right|^{1 / 2} .
$$

Remarquons que si on se restreint à une courbe complexe tangente joignant $z_{1}$ à $z_{2}$ la différentielle $d_{z} \Phi$ s'annule à l'ordre 1 en $z=\zeta$. Par des estimations analogues, on en déduit que $\tilde{R}_{M} f$ est de classe $\mathcal{C}^{1-\varepsilon}$ sur toute courbe complexe tangente de $M$.

Dans le cas des petits degrés, les rôles de $z$ et $\zeta$ sont inversés; pour appliquer les estimations ci-dessus nous avons donc besoin d'un noyau de classe $\mathcal{C}^{1}$ en $\zeta$. Comme $R_{M}$ fait intervenir des dérivées d'ordre 2 en $\zeta$ des fonctions définissantes de $M$, nous devons supposer que $M$ est de classe $\mathcal{C}^{3}$.

ThÉORÈme 5.2. Si $M$ est de classe $\mathcal{C}^{3}$ et $0 \leq r \leq q$, l'opérateur $\tilde{R}_{M}$ est continu de $W_{n, r}^{0, \infty}(\Omega)$ dans $\mathcal{C}_{n, r-1}^{1 / 2}(\Omega)$. 


\subsection{Estimations d'Ordre Supérieur}

Nous allons utiliser des idées développées dans [18] et reprises par [9].

On note $\Phi_{j}(z, \zeta)$ la fonction $\Phi_{J}$ pour $J=\left(\lambda_{1}, \ldots, \lambda_{k}, \lambda_{*}\right) \in \mathcal{I}^{\prime}(k *)$ avec $\lambda_{j}=1$.

LeMme 5.3. Il existe des champs de vecteurs $Y_{1}^{\zeta}, \ldots, Y_{k}^{\zeta}$ tangents à $M$ tels que pour tout $\zeta \in U\left(z_{0}\right)$ et $1 \leq i, j \leq k$

$$
Y_{i}^{\zeta} \Phi_{j}(\zeta, \zeta)=\delta_{i j}
$$

où $\delta_{i j}$ désigne le symbole de Kronecker.

Démonstration. Puisque $M$ est générique, $\partial \rho_{1} \wedge \cdots \wedge \partial \rho_{k} \neq 0$ sur $U\left(z_{0}\right)$ et par conséquent la matrice

où

$$
A=\left(\begin{array}{ccc}
\left\langle\partial \rho_{1}(\zeta), \partial \rho_{1}(\zeta)\right\rangle & \ldots & \left\langle\partial \rho_{k}(\zeta), \partial \rho_{1}(\zeta)\right\rangle \\
\vdots & & \vdots \\
\left\langle\partial \rho_{1}(\zeta), \partial \rho_{k}(\zeta)\right\rangle & \ldots & \left\langle\partial \rho_{k}(\zeta), \partial \rho_{k}(\zeta)\right\rangle
\end{array}\right),
$$

$$
\left\langle\partial \rho_{i}(\zeta), \partial \rho_{j}(\zeta)\right\rangle=\sum_{\nu=1}^{n} \frac{\partial \rho_{j}}{\partial \bar{\zeta}_{\nu}}(\zeta) \frac{\partial \rho_{i}}{\partial \zeta_{\nu}}(\zeta)
$$

est inversible pour tout $\zeta \in U\left(z_{0}\right)$ et il existe $v_{1}, \ldots, v_{k} \in\{1, \ldots, n\}$ tels que la matrice

$$
B=\left(\begin{array}{ccc}
\frac{\partial \rho_{1}}{\partial \zeta_{v_{1}}}(\zeta) & \cdots & \frac{\partial \rho_{k}}{\partial \zeta_{\nu_{1}}}(\zeta) \\
\vdots & & \vdots \\
\frac{\partial \rho_{1}}{\partial \zeta_{v_{k}}}(\zeta) & \cdots & \frac{\partial \rho_{k}}{\partial \zeta_{v_{k}}}(\zeta)
\end{array}\right)
$$

est inversible pour tout $\zeta \in U\left(z_{0}\right)$.

On pose

$$
Y_{i}^{\zeta}=\frac{1}{2} \sum_{j=1}^{k} \alpha_{i j}(\zeta) \sum_{v=1}^{n} \frac{\partial \rho_{j}}{\partial \bar{\zeta}_{v}}(\zeta) \frac{\partial}{\partial \zeta_{v}}-\frac{1}{2} \sum_{j=1}^{k} \beta_{i j}(\zeta) \frac{\partial}{\partial \bar{\zeta}_{v_{j}}}
$$

où $\left[\alpha_{i j}\right]=A^{-1}$ et $\left[\beta_{i j}\right]=B^{-1}$.

On montre alors facilement que pour $1 \leq i, j \leq k$

$$
Y_{i}^{\zeta} \Phi_{j}(\zeta, \zeta)=\delta_{i j} \quad \text { et } \quad Y_{i}^{\zeta} \rho_{j}=0 .
$$

Puisque $\rho_{*}=\rho_{1}+\cdots+\rho_{k}$, on a par définition de $\Phi_{I *}$

$$
\Phi_{I *}(z, \zeta, \lambda)=\sum_{j=1}^{k}\left(\lambda_{J}+\lambda_{*}\right) \Phi_{j}(z, \zeta)+O_{2} .
$$

Pour $\lambda \in \Delta_{I *}$, on définit le champs de vecteurs $Y_{\lambda}^{\zeta}$ par 


$$
Y_{\lambda}^{\zeta}=\sum_{j=1}^{k} \frac{\lambda_{j}+\lambda_{*}}{\sum_{j=1}^{k}\left(\lambda_{j}+\lambda_{*}\right)^{2}} Y_{j}^{\zeta}
$$

Le champs de vecteur $Y_{\lambda}^{\zeta}$ est tangent à $M$ et vérifie $Y_{\lambda}^{\zeta} \Phi_{I *}(\zeta, \zeta, \lambda)=1$ pour $\zeta \in$ $U\left(z_{0}\right)$ et $\lambda \in \Delta_{I *}$.

DÉfinition 5.4. Une forme différentielle définie sur $U\left(z_{0}\right) \times U\left(z_{0}\right) \times \Delta_{I *}$ appartient à la classe $\mathcal{E}_{\delta}$ si elle peut s'écrire

$$
\frac{E^{\alpha+k-m}}{\left(\Phi_{I *}+\delta\right)^{\beta}} \partial \rho_{i_{1}} \wedge \cdots \wedge \partial \rho_{i_{m}},
$$

où $E^{j}=O_{0}(\zeta-z)^{j}$ et $\alpha$ et $\beta$ sont des entiers tels que $2 n-1-2 \beta+\alpha \geq 0$.

On dira qu'un noyau $L_{\delta}$ est dans la classe $\mathcal{L}_{\delta}$ si

où $E_{\delta}$ appartient à $\mathcal{E}_{\delta}$.

$$
L_{\delta}(z, \zeta)=\int_{\lambda \in \Delta_{I *}} E_{\delta}(z, \zeta, \lambda)
$$

Le noyau $R_{M}$ est une somme finie d'éléments de la classe $\mathcal{L}_{0}$. Remarquons que si on remplace $R_{M}$ par un noyau de la classe $\mathcal{L}_{\delta}, \delta>0$, l'inégalité (5.4) reste vraie avec une constante $C$ qui ne dépend pas de $\delta, \operatorname{car}\left|\Phi_{I *}+\delta\right| \geq \frac{1}{2}\left(\operatorname{Re} \Phi_{I *}+\left|\operatorname{Im} \Phi_{I *}\right|\right)$.

Si $X^{z}$ est un champs de vecteur tangent à $M$ en la variable $z$, on note $X^{\zeta}$ le champs correspondant en la variable $\zeta$.

LEMME 5.5. Fixons $\delta>0$, pour tout noyau $E_{\delta}$ de classe $\mathcal{C}^{l}$ appartenant à $\mathcal{E}_{\delta}$, il existe $\varepsilon>0$ tel que pour $(z, \zeta) \in U\left(z_{0}\right) \times U\left(z_{0}\right)$ vérifiant $|z-\zeta|<\varepsilon$ on a

$$
X^{z} E_{\delta}=-X^{\zeta} E_{\delta}+\frac{\left(X^{z}+X^{\zeta}\right) \Phi_{I *}}{Y_{\lambda}^{\zeta} \Phi_{I *}} Y_{\lambda}^{\zeta} L_{\delta}+G_{\delta},
$$

où $G_{\delta}$ est une somme finie de noyaux de classe $\mathcal{C}^{l-1}$ appartenant à $\mathcal{E}_{\delta}$.

Démonstration. On remarque que puisque $Y_{\lambda}^{\zeta} \Phi_{I *}(\zeta, \zeta, \lambda)=1$ pour $\zeta \in U\left(z_{0}\right)$, il existe $\varepsilon>0$ et une constante $C$ telle que $Y_{\lambda}^{\zeta} \Phi_{I *}(z, \zeta, \lambda)>C$ si $|z-\zeta|<\varepsilon$. Par ailleurs

$$
\begin{aligned}
\left(X^{z}+X^{\zeta}\right) E^{j} & =E^{j}, \\
\left(X^{z}+X^{\zeta}\right) \Phi_{I *} & =E^{1}, \\
Y_{\lambda}^{\zeta} E^{j} & =E^{j-1} .
\end{aligned}
$$

Le lemme résulte alors d'un calcul direct.

On note $W_{n, r}^{l, \infty}(\Omega)$ l'espace des $(n, r)$-formes différentielles de classe $\mathcal{C}^{l}$ dont les dérivées d'ordre $l$ des coefficients appartiennent à $L^{\infty}(\Omega)$ et $\mathcal{C}_{n, r}^{l+1 / 2}(\Omega)$ l'espace des $(n, r)$-formes différentielles de classe $\mathcal{C}^{l}$ dont les dérivées d'ordre $l$ des coefficients sont hölderiennes d'ordre $1 / 2$.

THÉORÈME 5.6. Si $M$ est de classe $\mathcal{C}^{l+2}$ et $n-k-q+1 \leq r \leq n-k$, l'opérateur $\tilde{R}_{M}$ est continu de $W_{n, r}^{l, \infty}(\Omega)$ dans $\mathcal{C}_{n, r-1}^{l+1 / 2}(\Omega)$. 
Démonstration. Fixons un point $z_{1}$ dans $\Omega$ et choisissons une fonction $\chi$ à support compact dans $\Omega$ telle que $\chi(\zeta)=1$, si $\left|\zeta-z_{1}\right| \leq \varepsilon / 4$, et $\chi(\zeta)=0$, si $\left|\zeta-z_{1}\right| \geq$ $\varepsilon / 2$, où $\varepsilon$ est donné par le Lemme 5.5. On pose $D\left(z_{1}\right)=\left\{z \in \Omega|| z-z_{1} \mid \leq \varepsilon / 4\right\}$. On écrit

$$
\tilde{R}_{M} f(z)=\int_{\zeta \in \Omega} \chi(\zeta) f(\zeta) \wedge R_{M}(z, \zeta)+\int_{\zeta \in \Omega}(1-\chi(\zeta)) f(\zeta) \wedge R_{M}(z, \zeta)
$$

On note $J_{1}(f)$ la première intégrale du second membre et $J_{2}(f)$ la seconde.

Puisque $R_{M}(z, \zeta)$ est de classe $\mathcal{C}^{\infty}$ en $z$ pour $z \neq \zeta$, l'intégrale $J_{2}(f)$ est de classe $\mathcal{C}^{\infty}$ sur $D\left(z_{1}\right)$.

LEMME 5.7. Soient $\delta>0, L_{\delta}$ un noyau de classe $\mathcal{C}^{l}$ appartenant à $\mathcal{L}_{\delta}$ et $f$ une $(n, r)$-forme dans $W_{n, r}^{l, \infty}(\Omega)$. Si

$$
\tilde{L}_{\delta} f(z)=\int_{\zeta \in \Omega} \chi(\zeta) f(\zeta) \wedge L_{\delta}(z, \zeta)
$$

et si $X_{1}^{z}, \ldots, X_{j}^{z}$ sont des champs de vecteurs tangents à $M, j \leq l$, alors $X_{1}^{z} \ldots$ $X_{j}^{z} \tilde{L}_{\delta} f$ appartient à $\mathcal{C}_{n, r}^{1 / 2}(\Omega)$ et il existe une constante $C_{j}$ indépendante de $\delta$ et de $f$ telle que

$$
\left\|X_{1}^{z} \ldots X_{j}^{z} \tilde{L}_{\delta} f\right\|_{1 / 2, D\left(z_{1}\right)} \leq C_{j}\|f\|_{\infty} .
$$

Démonstration. Le Lemme 5.5 et une intégration par parties donnent

$$
X^{z} \tilde{L}_{\delta} f(z)=\int_{\zeta \in \Omega} \tilde{X}^{\zeta}(\chi(\zeta) f(\zeta)) \wedge L_{\delta}(z, \zeta)+\int_{\zeta \in \Omega} \chi(\zeta) f(\zeta) \wedge P_{\delta}(z, \zeta),
$$

où $\tilde{X}^{\zeta}$ est un champs de vecteurs tangent à $M$ indépendant de $\delta$ et $P_{\delta}$ une somme finie de noyaux de classe $\mathcal{C}^{l-1}$ appartenant à $\mathcal{L}_{\delta}$. En appliquant successivement les champs de vecteurs $X_{1}^{z}, \ldots, X_{j}^{z}$, on obtient donc

$$
X_{1}^{z} \ldots X_{j}^{z} \tilde{L}_{\delta} f(z)=\sum_{\nu=0}^{j} \sum_{j_{\nu} \in\{1, \ldots, j\}} \int_{\zeta \in \Omega} X_{j_{1}}^{\zeta} \ldots X_{j_{\nu}}^{\zeta} \chi(\zeta) f(\zeta) \wedge Q_{\delta}(z, \zeta),
$$

où $Q_{\delta}$ est une somme finie de noyaux de classe $\mathcal{C}^{l-1}$ appartenant à $\mathcal{L}_{\delta}$. On peut estimer les intégrales du membre de droite comme dans la Section 5.1 avec des constantes indépendantes de $\delta$ ainsi que nous l'avons remarqué après la Définition 5.4.

Terminons la démonstration du Théorème 5.6. On note $L_{\delta, M}$ le noyau obtenu en remplaçant $\Phi_{I *}$ par $\Phi_{I *}+\delta$ dans $R_{M}$. On peut montrer en utilisant les mêmes arguments que dans le Lemme 2.11 de [9] que $\tilde{L}_{\delta, M} f$ et $X_{1}^{z} \ldots X_{j}^{z} \tilde{L}_{\delta, M} f$ converge uniformément vers $\tilde{R}_{M} f$ et $X_{1}^{z} \ldots X_{j}^{z} \tilde{R}_{M} f$ sur $\Omega$ lorsque $\delta$ tend vers 0 . Les constantes $C_{j}$ du Lemme 5.7 étant indépendantes de $\delta$, le théorème est alors démontré.

Un travail similaire en inversant les rôles de $z$ et $\zeta$ permet d'obtenir le théorème suivant: 
THÉoRÈme 5.8. Si $M$ est de classe $\mathcal{C}^{l+3}$ et $1 \leq r \leq q$, l'opérateur $\tilde{R}_{M}$ est continu de $W_{n, r}^{l, \infty}(\Omega)$ dans $\mathcal{C}_{n, r-1}^{l+1 / 2}(\Omega)$.

Nous terminons en prouvant un lemme de Poincaré pour le $\bar{\partial}_{b}$ avec régularité $\mathcal{C}^{l+1 / 2}$.

THÉORÈME 5.9. Soient $M$ une variété CR générique q-concave, de codimension réelle $k$, de classe $\mathcal{C}^{3}$ plongée dans $\mathbb{C}^{n}$ et $z_{0}$ un point de $M$. Pour tout voisinage ouvert $U$ de $z_{0}$ dans $M$, il existe un voisinage ouvert $V \subset U$ de $z_{0}$ et pour tout $r$ tel que $1 \leq r \leq q-1$ ou $n-k-q+1 \leq r \leq n-k$, un opérateur

$$
T_{r}: \mathcal{C}_{n, r}(U) \rightarrow \mathcal{C}_{n, r}(V)
$$

possédant les propriétés suivantes:

(i) $\left.f\right|_{V}=\bar{\partial}_{b} T_{r} f+T_{r+1} \bar{\partial}_{b} f$, pour $1 \leq r \leq q-2$ et $n-k-q+1 \leq r \leq n-k$;

(ii) $\left.f\right|_{V}=\bar{\partial}_{b} T_{r} f$, si $r=q-1$ et $\bar{\partial}_{b} \bar{f}=\overline{0}$;

(iii) si $f \in \mathcal{C}_{n, r}^{l}(U)$ alors $T_{r} f \in \mathcal{C}_{n, r}^{l+1 / 2}(V)$ si $M$ est de classe $\mathcal{C}^{l+3}$ et $1 \leq r \leq$ $q-1$ ou si $M$ est de classe $\mathcal{C}^{l+2}$ et $n-k-q+1 \leq r \leq n-k$.

Démonstration. Sans perte de généralité on peut supposer que $U$ est contenu dans $M \cap U_{z_{0}}$. Soit $B$ une petite boule centrée en $z_{0}$ telle que $\Omega=B \cap M$ soit un domaine à bord $\mathcal{C}^{1}$ relativement compact dans $U$. Si $f$ est une $(n, r)$-forme continue sur $U$, on définit les opérateurs $P_{r}$ par:

$$
\begin{aligned}
& P_{r} f(\zeta)=(-1)^{(k+1)(r+n)+k(k+1) / 2} \int_{z \in b \Omega} f(z) \wedge\left[R_{M}\right]_{n, n-k-r-1}(z, \zeta) \\
& \text { si } 1 \leq r \leq q-1 \\
& P_{r} f(z)=(-1)^{(k+1)(r+n)+k(k+1) / 2} \int_{\zeta \in b \Omega} f(\zeta) \wedge\left[R_{M}\right]_{n, r}(z, \zeta) \\
& \text { si } n-k-q+1 \leq r \leq n-k .
\end{aligned}
$$

Il résulte de la définition des noyaux $R_{M}$ que les opérateurs $P_{r}$ sont continus de $\mathcal{C}_{n, r}^{l}(U)$ dans $\mathcal{C}_{n, r}^{l}(\Omega)$ si $M$ est de classe $\mathcal{C}^{l+3}$ et $1 \leq r \leq q-1$ ou si $M$ est de classe $\mathcal{C}^{l+2}$ et $n-k-q+1 \leq r \leq n-k$. Soit $B^{\prime}$ une autre boule centrée en $z_{0}$ telle que $B^{\prime} \cap M \subset \subset \Omega$. En utilisant le noyau de Henkin associé à la boule $B^{\prime}$, on obtient un opérateur linéaire continu $L_{r}$ de $\mathcal{C}_{n, r}^{l}\left(B^{\prime}\right)$ dans $\mathcal{C}_{n, r-1}^{l+\varepsilon}\left(B^{\prime}\right), 0<\varepsilon<1$, tel que

$$
\left.P_{r} f\right|_{B^{\prime}}=\bar{\partial} L_{r-1} P_{r} f+L_{r} \bar{\partial} P_{r} f .
$$

On pose alors

$$
T_{r} f=(-1)^{(k+1)(r+n)+k(k+1) / 2} \tilde{R}_{M} f+(-1)^{k} L_{r-1} P_{r} f .
$$

La formule d'homotopie du (i) résulte alors du Corollaire 4.3 et du fait que, grâce à (4.7), $\bar{\partial} P_{r}=P_{r+1} \bar{\partial}_{b}$, si $0 \leq s \leq q-2$ et $n-k-q+1 \leq r \leq n-k$.

Le point (ii) se déduit aussi du Corollaire 4.3 en remarquant que $\overline{\bar{\partial}} P_{r} f=0$, si $\bar{\partial}_{b} f=0$ et $r=q-1$. En appliquant la Proposition 3.2 et le Lemme 4.1 on obtient 


$$
\bar{\partial}_{z, \zeta} R_{M}=(-1)^{k+1} \sum_{I \in \mathcal{I}^{\prime}(k)} \operatorname{sgn}(I) C_{I} .
$$

Mais, d'après le Lemme 3.4, $\bar{\partial}_{z}\left[C_{I}\right]_{n, n-k-q}=0$ et par des arguments analogues à ceux développés dans [15, Lemme 5.4 et 5.5], pour tout $I \in \mathcal{I}^{\prime}(k)$, on peut approcher uniformément au voisinage de $b \Omega$ les $\left[C_{I}\right]_{n, n-k-q}$ par des formes $\bar{\partial}_{z^{-}}$ fermées au voisinage de $\bar{\Omega}$. Alors la formule de Stokes permet de prouver que $\bar{\partial} P_{q-1} f=0$, si $\bar{\partial}_{b} f=0$.

Finalement (iii) est une conséquence des Théorèmes 5.6 et 5.8 et de la régularité des opérateurs $L_{r}$.

\section{Appendice}

Cet appendice reprend des résultats démontrés dans [16], utilisés pour estimer l'intégration en $\lambda$ pour les différents noyaux.

Soient $h \geq 2$ un entier et $K=\left(k_{1}, \ldots, k_{s}\right) \in P^{\prime}(h)$, on pose $d \lambda_{K}=d \lambda_{k_{2}} \wedge$ $\cdots \wedge d \lambda_{k_{s}}$. Soient de plus $C_{*}, \delta$ et $\varepsilon$ des constantes positives, $\varphi_{1}, \ldots, \varphi_{h}$ des nombres complexes tels que

$$
\operatorname{Re} \varphi_{j} \geq \delta+\varepsilon
$$

Si $i, j \in\{1, \ldots, h\}$ avec $i \neq j, \nabla_{j}^{i}$ représente la dérivée partielle $\frac{\partial}{\partial \lambda_{j}}$ en considérant $\lambda_{j}$ dans le système de coordonnées $\left(\lambda_{1}, \ldots, \hat{\lambda}_{i}, \ldots, \lambda_{h}\right)$ sur $\Delta_{(1, \ldots, h)}$ et on note

$$
\nabla_{j_{1} \ldots j_{t}}^{i_{1} \ldots i_{t}}=\nabla_{j_{1}}^{i_{1}} \ldots \nabla_{j_{t}}^{i_{t}}
$$

pour $t \geq 2$, et $1 \leq i_{v}, j_{v} \leq h$, avec $i_{v} \neq j_{v}(v=1, \ldots, t)$.

Soient $\gamma$ et $\Gamma$ deux fonctions de classe $\mathcal{C}^{\infty}$ sur $\Delta_{(1, \ldots, h)}$ telles que

$$
\begin{aligned}
|\gamma(\lambda)| & \leq \frac{\varepsilon}{4}, \\
\left|\nabla_{j_{1} \ldots j_{t}}^{i_{1} \ldots i_{t}} \gamma(\lambda)\right| & \leq \frac{\varepsilon}{4}, \\
|\Gamma(\lambda)| & \leq C_{*}, \\
\left|\nabla_{j_{1} \ldots j_{t}}^{i_{1} \ldots i_{t}} \Gamma(\lambda)\right| & \leq C_{*},
\end{aligned}
$$

pour tout $\lambda \in \Delta_{(1, \ldots, h)}, 1 \leq t \leq h+2,1 \leq i_{v}, j_{v} \leq h$, avec $i_{v} \neq j_{v}(v=1, \ldots, t)$.

Rappelons les estimations prouvées dans le Théorème 6.1 de [16]:

THÉORÈME 6.1. Si pour tout $p \in \mathbb{N}$, on note $C_{p}=(3 p) ! 2^{7 p}$, alors:

1. pour tout $p \in \mathbb{N}$, on a

$$
\left|\int_{\Delta_{(1, \ldots, h)}} \frac{\Gamma(\lambda) d \lambda_{(1, \ldots, h)}}{\left(\sum_{j=1}^{h} \lambda_{j} \varphi_{j}+\gamma(\lambda)\right)^{p}}\right| \leq \frac{C_{p} C_{*}}{(\delta+\varepsilon)^{p}} ;
$$

2. pour tout $K \in P^{\prime}(h)$ et tout $p \geq|K|+1$,

$$
\left|\int_{\Delta_{(1, \ldots, h)}} \frac{\Gamma(\lambda) d \lambda_{(1, \ldots, h)}}{\left(\sum_{j=1}^{h} \lambda_{j} \varphi_{j}+\gamma(\lambda)\right)^{p}}\right| \leq \frac{C_{p} C_{*}}{\prod_{j \in K}\left|\varphi_{j}\right|(\delta+\varepsilon)^{p-|K|}} .
$$


On définit maintenant la notion de famille de sommets admissibles correspondant à la Définition 7.3 dans [16]:

DÉfinition 6.2. Soit $\alpha$ la constante donnée par la relation (3.5). Une famille de sommets admissibles est une famille ordonnée $\left(\lambda^{1}, \ldots, \lambda^{l}\right)$ de points de $\Delta_{(1, \ldots, N+1)}$ telle que:

(i) il existe $K=\left(k_{1}, \ldots, k_{l}\right) \in P^{\prime}(N+1)$ tel que $\lambda^{j} \in \Delta_{K}$, pour $j=1, \ldots, l$;

(ii) $\lambda_{1}, \ldots, \lambda_{l}$ sont des vecteurs linéairement indépendants dans $\mathbb{R}^{l}$;

(iii) pour tout $(\zeta, z, \tau) \in \mathbb{C}^{n} \times U_{\bar{D}} \times \Delta_{(1, \ldots, l)}$ la fonction $\gamma$ définie par

$$
\gamma(\zeta, z, \tau)=\varphi\left(\zeta, z, \sum_{j=1}^{l} \tau_{j} \lambda^{j}\right)-\sum_{j=1}^{l} \tau_{j} \varphi\left(\zeta, z, \lambda^{j}\right)
$$

vérifie les relations suivantes:

$$
\begin{aligned}
|\gamma(\zeta, z, \tau)| & \leq \frac{\alpha}{8}|\zeta-z|^{2}, \\
\left|\nabla_{j_{1} \ldots j_{t}}^{i_{1} \ldots i_{t}} \gamma(\zeta, z, \tau)\right| & \leq \frac{\alpha}{8}|\zeta-z|^{2},
\end{aligned}
$$

pour tout $1 \leq t \leq l+2,1 \leq i_{v}, j_{v} \leq l$, avec $i_{v} \neq j_{v}(v=1, \ldots, t)$.

Si $\left(\lambda^{1}, \ldots, \lambda^{l}\right)$ est une famille de sommets admissibles, on note

$$
\Delta\left(\lambda^{1}, \ldots, \lambda^{l}\right)=\left\{\sum_{j=1}^{l} \tau_{j} \lambda^{j}, \tau \in \Delta_{1, \ldots, l}\right\} .
$$

On dit alors qu'un simplexe $\Delta$ est admissible s'il existe une famille de sommets admissibles, $\left(\lambda^{1}, \ldots, \lambda^{l}\right)$, telle que $\Delta=\Delta_{\lambda^{1}, \ldots, \lambda^{l}}$.

On peut maintenant énoncer le lemme suivant, qui est démontré dans [16]:

Lemme 6.3. Il existe $\varepsilon>0$ tel que, si $K=\left(k_{1}, \ldots, k_{l}\right) \in P^{\prime}(N+1)$ et $\lambda^{1}, \ldots, \lambda^{l} \in$ $\Delta_{K}$ sont des vecteurs linéairement indépendants avec

$$
\left|\lambda^{i}-\lambda^{j}\right|<\varepsilon \quad(1 \leq i, j \leq l)
$$

alors $\left(\lambda^{1}, \ldots, \lambda^{l}\right)$ est une famille de sommets admissibles.

D'après le Lemme 6.3, on peut diviser $\Delta_{I}$ en un nombre fini de simplexes admissibles.

Si $\lambda^{1}, \ldots, \lambda^{l}$ est une famille de sommets admissible fixée, on pose

$$
\tilde{\Delta}\left(\lambda^{1}, \ldots, \lambda^{l}\right)=\left\{\lambda \in \stackrel{\circ}{\Delta}_{0 I} \mid \stackrel{\circ}{\lambda} \in \Delta\left(\lambda^{1}, \ldots, \lambda^{l}\right)\right\} .
$$

Les intégrales sur $\Delta_{0 I}$ sont donc des sommes finies d'intégrales associées à des simplexes admissibles. 
Pour majorer l'intégration en $\lambda$, on remarque alors que

$$
\begin{aligned}
\psi:\left[0, \frac{1}{2}\right] \times \Delta_{I} & \longrightarrow \tilde{\Delta}, \\
(\tau, \vartheta) & \longmapsto\left(\tau,(1-\tau) \sum_{j=1}^{l} \vartheta_{j} \lambda_{1}^{j}, \ldots,(1-\tau) \sum_{j=1}^{l} \vartheta_{j} \lambda_{l}^{j}\right),
\end{aligned}
$$

est un difféomorphisme et on peut alors utiliser le Théorème 6.1.

\section{Références}

[1] R. A. Airapetjan et G. M. Henkin, Integral representation of differential forms on Cauchy-Riemann manifolds and the theory of CR function, Russian Math. Surveys 39 (1984), 41-118.

[2] - Integral representation of differential forms on Cauchy-Riemann manifolds and the theory of CR function II, Mat. Sb. (N.S.) 127 (1985), 92-112.

[3] M. Y. Barkatou, Formules locales de type Bochner-Martinelli-Koppelman sur des variétés $C R$, applications, Thèse, Grenoble, 1994.

[4] - Régularité hölderienne du $\bar{\partial}_{b}$ sur les hypersurfaces 1-convexes-concaves, Math. Z. 221 (1996), 549-572.

[5] - Some applications of a new integral formula for $\bar{\partial}_{b}$, Ann. Polon. Math. 70 (1998), 1-24.

[6] - Optimal regularity for $\bar{\partial}_{b}$ on CR manifolds, J. Geom. Anal. 10 (2000), 1-23.

[7] A. Boggess, CR manifolds and the tangential Cauchy-Riemann complex, CRC Press, Boca Raton, FL, 1991.

[8] A. Boggess et M.-C. Shaw, A kernel approach to the local solvability of the tangential Cauchy-Riemann equations, Trans. Amer. Math. Soc. 289 (1985), 643-659.

[9] B. Fischer, Kernels of Martinelli-Bochner type on hypersurfaces, Math. Z. 223 (1996), 155-183.

[10] B. Fischer et J. Leiterer, A local Martinelli-Bochner formula on hypersurfaces, Math. Z. 214 (1993), 659-681.

[11] R. Harvey et J. Polking, Fundamental solutions in complex analysis, Part I and II, Duke Math. J. 46 (1979), 253-300 and 301-340.

[12] G. M. Henkin, The Hans Lewy equation and analysis on pseudoconvex manifolds, Russian Math. Surveys 321 (1977), 59-130.

[13] - Solution des équations de Cauchy-Riemann tangentielles sur des variétés Cauchy-Riemann q-concaves, C. R. Acad. Sci. Paris Sér. I Math. 292 (1981), 27-30.

[14] G. M. Henkin et J. Leiterer, Andreotti-Grauert theory by integral formulas, Progr. Math., 74, Birkhäuser, Boston, 1988.

[15] C. Laurent-Thiébaut et J. Leiterer, Uniform estimates for the Cauchy-Riemann equation on q-concave wedges, Colloque d'analyse complexe et géométrie (Marseille, 1992), Astérisque 217 (1993), 151-182.

[16] - Uniform estimates for the Cauchy-Riemann equation on q-convex wedges, Ann. Inst. Fourier (Grenoble) 43 (1993), 383-436.

[17] - On Polyakov's notion of regular q-concave CR manifold, Math. Z. 253 (2006), 235-249.

[18] L. Ma et J. Michel, Local regularity for the tangential Cauchy-Riemann, J. Reine Angew. Math. 442 (1993), 63-90. 
[19] P. L. Polyakov, Sharp estimates for operator $\bar{\partial}_{M}$ on a q-concave CR manifold, J. Geom. Anal. 6 (1996), 233-276.

[20] - Global $\bar{\partial}_{M}$-homotopy with $\mathcal{C}^{k}$ estimates for a family of compact, regular q-pseudoconcave CR manifolds, Math. Z. 247 (2004), 813-862.

[21] A. V. Romanov, A formula and estimates for the solution of the tangential CauchyRiemann equation, Mat. Sb. (N.S.) 99 (1976) 58-83.

[22] M.-C. Shaw, Integral representations for $\bar{\partial}_{b}$ in CR manifolds, Geometric complex analysis (Hayama, 1995), pp. 535-549, World Scientific, River Edge, NJ, 1996.

[23] H. Skoda, Valeurs au bord pour les solutions de l'opérateur d et caractérisation des zéros des fonctions de la classe de Nevanlinna, Bull. Soc. Math. France 104 (1976), 225-299.

M. Y. Barkatou

Université de Poitiers SP2MI

UMR 6086 Groupes de Lie et Géométrie

Bd Marie et Pierre Curie

BP 30179

86962 Futuroscope Chasseneuil Cedex

France

barkatou@mathlabo.univ-poitiers.fr
C. Laurent-Thiébaut

Université de Grenoble

Institut Fourier

UMR 5582 CNRS/UJF

BP 74

38402 St Martin d'Hères Cedex

France

Christine.Laurent@ujf-grenoble.fr 